\title{
Born-Infeld gravity coupled to Born-Infeld electrodynamics
}

\author{
Soumya Jana and Sayan Kar * \\ Department of Physics and Centre for Theoretical Studies \\ Indian Institute of Technology, Kharagpur, 721302, India
}

We investigate spherically symmetric, static spacetimes in Eddington-inspired Born-Infeld gravity coupled to Born-Infeld electrodynamics. The two constants, $b^{2}$ and $\kappa$ which parametrise the BornInfeld structures in the electrodynamics (matter) and gravity sectors, characterise the features of our analytical solutions. Black holes or naked singularities are found to arise, depending on the values of $b^{2}$ and $\kappa$, as well as charge and mass. Several such solutions are classified and understood through the analysis of the associated metric functions for fixed $\kappa$, varying $b^{2}$ and vice-versa. Further, we also compare the new metric functions with those for the known $b^{2} \rightarrow \infty$ (Maxwell) and the $\kappa \rightarrow 0$ (geonic black hole) cases. Interestingly, for a particular relation between these two parameters, $b^{2}=1 / 4 \kappa, \kappa>0$, we obtain a solution resembling the well-known Reissner-Nordström line element, albeit some modifications. Using this particular solution as the background spacetime, we study null geodesics for Born-Infeld photons and also, gravitational lensing. Among interesting features we note $(i)$ an increase in the radius of the photon sphere with increasing $\kappa$ and (ii) a net positive contribution in the leading order correction term involving $\kappa$, in the weak lensing formula for the deflection angle. We also investigate the effective potential and light propagation for various other solutions through numerics and plots. In summary, our work is the first attempt towards figuring out how Born-Infeld structures in both the matter and gravity sectors can influence the nature and character of resulting gravitational fields.

* soumyajana@phy.iitkgp.ernet.in, sayan@iitkgp.ac.in 


\section{INTRODUCTION}

General Relativity (GR) is largely successful as a classical theory of gravity. It has been tested through experiments and observations to very high precision, in vacuum. However, it is not entirely certain that GR is the correct theory of gravity inside a matter distribution or, in the strong field regime. Moreover, it possesses many unsolved puzzles, both theoretical and observational. This has prompted many researchers to actively pursue various modified theories of gravity. For example, one motivation for constructing modified theories of gravity is to look for the possibility of avoiding the singularity problem in GR, eg. the occurrence of a big bang in cosmology or black holes in an astrophysical context. In a classical metric theory of gravity, under very general and physical assumptions, these singularities are inevitable, as proved through the Hawking-Penrose theorems [1]. However, it is possible to obtain non-singular geometries as solutions in an alternative theory. For example, non-singular Friedman-Robertson-Walker (FRW) type cosmologies do exist in modified gravity theories. One can also argue that the singularity in the classical theory is expected to be resolved in quantum gravity. Unfortunately, though specific examples exist in the context of loop quantum cosmology, we are still far away from any general statements which may be possible when we have a fully consistent quantum description of gravity.

In this article, we investigate one such alternate theory which is inspired from Eddington's alternative formulation of GR. In this formulation, the $\sqrt{-g} R$ (Ricci scalar) in the Einstein-Hilbert action is replaced by $\sqrt{-\operatorname{det}\left(R_{i j}\right)}$ and the connection is chosen as the basic variable, instead of the metric. Thus, Eddington's formulation is essentially an affine formulation [2]. In vacuum, it is equivalent to GR and it describes de Sitter gravity. However, coupling of matter was never addressed in this formulation until very recently.

We are also aware of Born-Infeld electrodynamics [3] wherein the divergence in the electric field at the location of the charge/current is removed by using an action different from that of Maxwell. The new field theory due to Born and Infeld includes the determinantal structures in the action, inspired by Eddington's work. The virtue of this nonlinear electrodynamics in removing the singularity of the electric field, inspired Deser and Gibbons to construct a Born-Infeld gravity theory in the metric formulation [4]. Later, Vollick [5] introduced the Palatini formulation in BornInfeld gravity and worked on various related aspects. He also introduced a non-trivial and somewhat artificial way of coupling matter in such a theory [6, 7]. More recently, Banados and Ferreira [8] have come up with a formulation where the matter coupling is different and simpler compared to Vollick's proposal. We will focus here on the theory proposed in [8] and refer to it as Eddington-inspired Born-Infeld (EiBI) gravity, for obvious reasons. Note that the EiBI theory has the feature that it reduces to GR, in vacuum.

Interestingly, EiBI theory also falls within the class of bimetric theories of gravity (also called bi-gravity). The current bimetric theories have their origin in the seminal work of Isham, Salam and Strathdee [9]. Several articles have appeared in the last few years on various aspects of such bi-gravity theories. In [10], the authors have pointed out that the EiBI field equations can also be derived from an equivalent bi-gravity action. This action is closely related to a recently discovered family of unitary massive gravity theories which are built as bi-gravity theories. Several others have contributed in this direction, in various ways [11, 12].

Let us now briefly recall Eddington-inspired Born-Infeld gravity. The central feature here is the existence of a physical metric which couples to matter and another auxiliary metric which is not used for matter couplings. One needs to solve for both metrics through the field equations. The action for the theory developed in [8], is given as:

$$
S_{B I}(g, \Gamma, \Psi)=\frac{1}{8 \pi \kappa} \int d^{4} x\left[\sqrt{-\left|g_{\mu \nu}+\kappa R_{\mu \nu}(\Gamma)\right|}-\lambda \sqrt{-g}\right]+S_{M}(g, \Psi)
$$

where $\Lambda=\frac{\lambda-1}{\kappa}$. We assume $G=1$ and $c=1$ throughout in the paper. Variation w.r.t $\Gamma$, done using the auxiliary metric $q_{\mu \nu}=g_{\mu \nu}+\kappa R_{\mu \nu}(q)$ gives

$$
q_{\mu \nu}=g_{\mu \nu}+\kappa R_{\mu \nu}(q)
$$

Variation w.r.t $g_{\mu \nu}$ gives

$$
\sqrt{-q} q^{\mu \nu}=\lambda \sqrt{-g} g^{\mu \nu}-8 \pi \kappa \sqrt{-g} T^{\mu \nu}
$$

In order to obtain solutions, we need to assume a $g_{\mu \nu}$ and a $q_{\mu \nu}$ with unknown functions, as well as a matter stress energy $\left(T^{\mu \nu}\right)$. Thereafter, we write down the field equations and obtain solutions using some additional assumptions about the metric functions and the stress energy.

Quite some work on various fronts have been carried out on various aspects of this theory, in the last few years. Astrophysical scenarios have been discussed in [13 21] while cosmology has been dealt with in 10, 22 29]. Among other topics, a domain wall brane in a higher dimensional generalisation, has been analysed in [30]. Generic features of the paradigm of matter-gravity couplings have been analysed in [31]. In [32], authors have shown that EiBI 
theory admits some nongravitating matter distribution, which is not allowed in GR. Some interesting cosmological and circularly symmetric solutions in $2+1$ dimensions are shown in [33]. Constraints on the EiBI parameter $\kappa$ are obtained from studies on compact stars [13, 17], tests in the solar system [34], astrophysical and cosmological observations [15], and nuclear physics studies [35]. In [36], a major problem related to surface singularities has been noticed in the context of stellar physics. However, gravitational backreaction has been suggested as a cure to this problem in [37]. In [38], the authors propose a modification to EiBI theory by taking its functional extension in a way similar to $f(R)$ theory. Recently, the authors in [39] use a different way of matter coupling, by taking the Kaluza ansatz for five dimensional EiBI action in a purely metric formulation and then compactify it using Kaluza's procedure to get a four dimensional gravity coupled in a nonlinear way to the electromagnetic theory.

Not much has however been done on black hole physics, or, broadly on the spherically symmetric, static solutions in this theory. It may be noted that the vacuum, spherically symmetric static solution is trivially same as the Schwarzschild de Sitter black hole. But, the electrovacuum solutions are expected to deviate from the usual ReissnerNordström solution in GR. This has been shown in [8, 40, 41] where the authors consider EiBI gravity coupled to a Maxwell electric field of a localized charge. They obtain the resulting spacetime geometries, and study its properties. The basic features of such spacetimes includes a singularity at the location of the charge which may or may not be covered by an event horizon. The strength of the electric field remains nonsingular as in Born-Infeld electrodynamics. However, this may not be the only solution because, in EiBI gravity, the matter coupling is nonlinear. The authors of [42] have shown, in a different framework, that the central singularity could be replaced by a wormhole supported by the electric field. In [43], the author obtained a class of Lorentzian regular wormhole spacetimes supported by the quintessential matter which does not violate the weak or null energy cindition in EiBI gravity.

In our present work, we couple EiBI gravity with Born-Infeld electrodynamics. Thus, we have Born-Infeld structures in both the gravity and matter sectors. We obtain some new classes of spherically symmetric static spacetimes and study their properties. The solutions we present include black holes and naked singularities. Earlier, a lot of work has indeed been done by considering nonlinear electrodynamics coupled to GR 44 51]. Some of them are motivated by string theory since Born-Infeld structures naturally arise in the low energy limit of open string theory [52, 53]. Our article is sectioned as follows. In Section II, we derive the form of the stress-energy tensor assuming an ansatz for the physical metric $\left(g_{\mu \nu}\right)$. We obtain the general expressions for the metric functions by solving the EiBI field equations with an ansatz for the auxiliary metric $\left(q_{\mu \nu}\right)$. In Section III, we list the solutions by scanning across regions in the parameter space $\left(b^{2}, \kappa\right)$, where $b^{2}$ and $\kappa$ are the parameters in the electromagnetic and gravity sectors, respectively. In Section IV, we analyze, in detail, a particular class of solutions of the Reissner-Nordström-type for which $b^{2}=\frac{1}{4 \kappa}$ $(\kappa>0)$. Here we also discuss null geodesics, photon propagation, and gravitational lensing. Finally, in Section V, we investigate the effective potential and gravitational lensing for the $b^{2} \neq \frac{1}{4 \kappa}$ solutions. In Section VI, we summarize our results and indicate possibilities for future work.

\section{SPHERICALLY SYMMETRIC STATIC SPACETIMES DUE TO A CHARGED MASS}

In curved spacetime, the Lagrangian density for the Born-Infeld electromagnetic field theory is given by [3],

$$
\mathcal{L}=\frac{b^{2} \sqrt{-g}}{4 \pi}\left[1-\sqrt{1+\frac{F}{b^{2}}-\frac{\mathcal{G}^{2}}{b^{4}}}\right]
$$

where, $F=\frac{1}{2} F_{\mu \nu} F^{\mu \nu}$ and $\mathcal{G}=\frac{1}{4} F_{\mu \nu} \mathcal{G}^{\mu \nu}$ are two scalar quantities constructed from the components of the electromagnetic field tensor $\left(F_{\mu \nu}\right)$ and the dual field tensor $\left(\mathcal{G}_{\mu \nu}\right)$. Here, $b$ sets an upper limit on the electromagnetic field and, when $b \rightarrow \infty$, Maxwell's theory is recovered. The resulting energy-momentum tensor has the following general expression:

$$
T_{\mu \nu}=-\frac{2}{\sqrt{-g}} \frac{\partial \mathcal{L}}{\partial g^{\mu \nu}}=-\frac{b^{2}}{4 \pi}\left[g_{\mu \nu}\left(\sqrt{1+\frac{F}{b^{2}}-\frac{\mathcal{G}^{2}}{b^{4}}}-1\right)-\frac{b^{2} F_{\mu \sigma} F_{\nu}^{\sigma}-\mathcal{G}^{2} g_{\mu \nu}}{b^{4} \sqrt{1+\frac{F}{b^{2}}-\frac{\mathcal{G}^{2}}{b^{4}}}}\right]
$$

In our work here we restrict ourselves to an electrostatic scenario (i.e. $A_{\mu} \equiv\{\phi, 0,0,0\}, \mathcal{G}=0$ ), in the background of a spherically symmetric, static spacetime. We assume the line element to be of the form,

$$
d s^{2}=-U(\bar{r}) e^{2 \psi(\bar{r})} d t^{2}+U(\bar{r}) e^{2 \nu(\bar{r})} d \bar{r}^{2}+V(\bar{r}) \bar{r}^{2}\left(d \theta^{2}+\sin ^{2} \theta d \phi^{2}\right)
$$

where, $\bar{r}$ is the radial coordinate, but the physical radial distance or, the area radius of each 2-sphere is $r=\bar{r} \sqrt{V(\bar{r})}$. With this assumption, solving the equations of motion for the electromagnetic field, we obtain

$$
\frac{d \phi}{d \bar{r}}=-\frac{q U e^{\nu+\psi}}{\sqrt{V^{2} \bar{r}^{4}+q^{2} / b^{2}}}
$$


were, $q$ is the electric charge. In the Minkowski limit of Eq. (6), the Eq. (7) resembles the known Born-Infeld electric field due to a point charge. The non-zero components of the energy-momentum tensor are given as:

$$
\begin{aligned}
T_{t t} & =\frac{b^{2} U}{4 \pi}\left(\frac{\sqrt{V^{2} \bar{r}^{4}+q^{2} / b^{2}}}{V \bar{r}^{2}}-1\right) e^{2 \psi}, \\
T_{\bar{r} \bar{r}} & =-\frac{b^{2} U}{4 \pi}\left(\frac{\sqrt{V^{2} \bar{r}^{4}+q^{2} / b^{2}}}{V \bar{r}^{2}}-1\right) e^{2 \nu}, \\
T_{\theta \theta} & =\frac{b^{2}}{4 \pi}\left(1-\frac{V \bar{r}^{2}}{\sqrt{V^{2} \bar{r}^{4}+q^{2} / b^{2}}}\right) V \bar{r}^{2}, \\
\text { and, } T_{\phi \phi} & =\frac{b^{2}}{4 \pi}\left(1-\frac{V \bar{r}^{2}}{\sqrt{V^{2} \bar{r}^{4}+q^{2} / b^{2}}}\right) V \bar{r}^{2} \sin ^{2} \theta .
\end{aligned}
$$

The energy-momentum tensor is not traceless ( i.e. $g^{\mu \nu} T_{\mu \nu} \neq 0$ for any $q / b \neq 0$ ). The auxiliary line element is assumed to be of the form

$$
d s_{q}^{2}=-e^{2 \psi} d t^{2}+e^{2 \nu} d \bar{r}^{2}+\bar{r}^{2}\left(d \theta^{2}+\sin ^{2} \theta d \phi^{2}\right)
$$

Here, the area radius of the auxiliary spacetime is $\bar{r}$. It is worth noting that our choice of the physical and auxiliary line elements are opposite to the standard choices in [8, 40 42]. While making this choice, we have exploited two facts: $(i)$ the absence of differential expressions in the field equations in (3) which opens up the scope of algebraic manipulations, and $(i i)$ the specific forms of the $t t$ - and $\bar{r} \bar{r}$ - components of the stress-energy tensor. This choice simplifies the situation to quite an extent. Using Eqs. (6), (8), and (9) in the field equations obtained from the ' $g$ 'variation [Eq. (3)], and after some algebra, we obtain the expressions for $U$ and $V$. We get a quadratic equation for $V$ as,

$$
\left(1-4 \kappa b^{2}\right) V^{2}-2\left(1-2 \kappa b^{2}\right) V+1-\frac{4 \kappa^{2} q^{2} b^{2}}{\bar{r}^{4}}=0
$$

There are two possible solutions for $V$ from Eq. (10) and consequently, two solutions for $U$. We choose the solution for $U$ and $V$ such that $U \rightarrow 1, V \rightarrow 1$ for large $\bar{r}$ and the physical spacetime is that found in the GR regime. Therefore, we have,

$$
\begin{aligned}
& V(\bar{r})=\frac{1-2 \kappa b^{2}\left(1+\sqrt{1+\frac{q^{2}}{b^{2} \bar{r}^{4}}-\frac{4 \kappa q^{2}}{\bar{r}^{4}}}\right)}{1-4 \kappa b^{2}} \\
& U(\bar{r})=\frac{\left(1-2 \kappa b^{2}\right) \sqrt{1+\frac{q^{2}}{b^{2} \bar{r}^{4}}-\frac{4 \kappa q^{2}}{\bar{r}^{4}}}-2 \kappa b^{2}}{\left(1-4 \kappa b^{2}\right) \sqrt{1+\frac{q^{2}}{b^{2} \bar{r}^{4}}-\frac{4 \kappa q^{2}}{\bar{r}^{4}}}}
\end{aligned}
$$

From the field equations obtained via ' $\Gamma$ '-variation [Eq. (2)], we get,

$$
\begin{gathered}
\kappa e^{-2 \psi} R_{t t}(q)-U=-1, \\
\kappa e^{-2 \nu} R_{\bar{r} \bar{r}}(q)+U=1, \\
\text { and } V \bar{r}^{2}+\kappa\left[-\frac{d}{d \bar{r}}\left(\bar{r} e^{-2 \nu}\right)+1\right]=\bar{r}^{2},
\end{gathered}
$$

where, $R_{t t}(q)=e^{-2 \nu+2 \psi}\left(\frac{2 \psi^{\prime}}{\bar{r}}-\nu^{\prime} \psi^{\prime}+\psi^{\prime 2}+\psi^{\prime \prime}\right)$ and $R_{\bar{r} \bar{r}}(q)=\frac{2 \nu^{\prime}}{\bar{r}}+\nu^{\prime} \psi^{\prime}-\psi^{\prime 2}-\psi^{\prime \prime}$. Here, primes denote derivatives with respect to $\bar{r}$. From Eq. (13) and Eq. (14), we get $\psi^{\prime}+\nu^{\prime}=0$. So, without loss of any generality, we can assume $\nu=-\psi$. Thus, from the Eq. (15), we arrive at,

$$
e^{2 \psi(\bar{r})}=1-\frac{\bar{r}^{2}}{3 \kappa}+\frac{1}{\kappa \bar{r}} \int V(\bar{r}) \bar{r}^{2} d \bar{r}+\frac{C}{\bar{r}}
$$

where, $C$ is a constant of integration. For, $q=0$ (i.e. vacuum), $U=V=1$ [Eqs. (12), (11)] for any $\kappa$, and the solution should be the Schwarzschild spacetime. Therefore, $C$ is identified as related to the total mass, $i . e ., C=-2 M$. 


\section{CLASSIFICATION OF SPACETIMES}

\section{A. General Solutions}

In this section, we obtain different classes of solutions by scanning the $b^{2}-\kappa$ parameter space. For this purpose, we introduce the definition $b^{2}=\frac{\alpha}{4 \kappa}$. We note that, if $\kappa>0$, then $\alpha>0$, and if $\kappa<0$ then $\alpha<0$. The characteristics/forms of the solutions change over different ranges of $\alpha$. These are-(i) $\alpha \rightarrow \infty,($ ii $) \infty>\alpha>1$, (iii) $\alpha \rightarrow 1$, (iv) $-\infty<\alpha<1$ (but $\neq 0$ ), and $(v) \alpha \rightarrow-\infty$. We discuss the $\alpha=1$ case elaborately, as a special case, in a separate section. The remaining classes of solutions are analysed here. Firstly, we note that $\alpha \rightarrow \pm \infty$ cases are actually those belonging to the Maxwell limit, i.e., a coupling of EiBI gravity with a Maxwellian electric field. This has already been studied earlier [40 42]. One can verify that the solution for this case (particularly for $\alpha \rightarrow \infty$ ), after rewriting in the Schwarzschild gauge, takes exactly the same form as that of [40]. Next, we obtain the solutions for the cases (ii) and (iv) below:

First, we rewrite $U, V$ as,

$$
\begin{aligned}
& U(\bar{r})=\frac{2-\alpha}{2(1-\alpha)}-\frac{\alpha}{2(1-\alpha)} \frac{1}{\sqrt{1+\frac{4 \kappa q^{2}(1-\alpha)}{\alpha \bar{r}^{4}}}}, \\
& V(\bar{r})=\frac{2-\alpha}{2(1-\alpha)}-\frac{\alpha}{2(1-\alpha)} \sqrt{1+\frac{4 \kappa q^{2}(1-\alpha)}{\alpha \bar{r}^{4}}} .
\end{aligned}
$$

Using, the Eq. (18), we compute the integral $\left(\int V \bar{r}^{2} d \bar{r}\right)$ (see the Appendix) and use it in the Eq. (16) to find the expression of $e^{2 \psi(\bar{r})}$.

(ii) $\infty>\alpha>1$ case:

Using Eq. A.3), we get,

$$
\begin{aligned}
e^{2 \psi(\bar{r})}= & 1+\frac{\alpha \bar{r}^{2}}{6 \kappa(\alpha-1)}\left[\sqrt{1-\frac{4 \kappa q^{2}(\alpha-1)}{\alpha \bar{r}^{4}}}-1\right]+\frac{\alpha^{1 / 4}\left(4 q^{2}\right)^{3 / 4}}{3 \kappa^{1 / 4}(\alpha-1)^{1 / 4} \bar{r}} F\left(\arcsin \left(\frac{\left(4 \kappa q^{2}(\alpha-1)\right)^{1 / 4}}{\alpha^{1 / 4} \bar{r}}\right) \mid-1\right) \\
& -\frac{2 M}{\bar{r}}
\end{aligned}
$$

where, $F(\phi \mid m)=\int_{0}^{\phi}\left[1-m \sin ^{2} \theta\right]^{-1 / 2} d \theta$ is the incomplete elliptic integral of the first kind. Thus we find all the metric functions. Here, the radius-squared of the 2 -sphere at each value of the radial coordinate $\bar{r}$ is given by $r^{2}(\bar{r})=V(\bar{r}) \bar{r}^{2}$. We note that, for $1<\alpha \leq 2$, at $\bar{r}=\left(\alpha \kappa q^{2}\right)^{1 / 4}$, the radius-square $\left(r^{2}\right)$ becomes zero. But, for $\alpha>2$, there is a non-zero minimum value of $r^{2}$, i.e. $r^{2}\left(\bar{r}_{0}\right)=\frac{(\alpha-2) \bar{r}_{0}^{2}}{2(\alpha-1)}$, where $\bar{r}_{0}=\left[4 \kappa q^{2}(1-1 / \alpha)\right]^{1 / 4}$. This means, for $1<\alpha \leq 2$, the spacetime is due to a point charge $(q)$ of total mass $M$, but, for $\alpha>2$, it is distributed over a spherical shell in space. One can verify that, for a very small value of $\kappa$, the solution converges to the known Reissner-Nordström solution. The reason for this is that, as $\kappa$ becomes very small with respect to the value of $\alpha, b^{2}$ becomes large, which then is the Maxwell limit for the electric field and the GR limit in the gravity sector. Further, we have examined the singularities of the spacetime and found that curvature scalar $(\mathcal{R})$ diverges at the position of the charge. Moreover, the existence of the horizon depends upon the values of the parameters $\alpha, \kappa, q$, and $M$. Some of these features are demonstrated in Fig. 1 and Fig. 3. where the plots of the metric functions in the Schwarzschild coordinates are shown.

In the Schwarzschild gauge, the line element [eq. [6] ] becomes

$$
\begin{aligned}
d s^{2} & =-g_{t t} d t^{2}+g_{r r} d r^{2}+r^{2}\left(d \theta^{2}+\sin ^{2} \theta d \phi^{2}\right) \\
\text { where }, \quad g_{t t} & =U(\bar{r}) e^{2 \psi(\bar{r})}, \quad g_{r r}=\frac{V(\bar{r})}{U(\bar{r})} e^{-2 \psi(\bar{r})}, \text { and }, \quad \bar{r}=r\left[1-\frac{\alpha}{2}+\frac{\alpha}{2} \sqrt{1+\frac{4 \kappa q^{2}}{\alpha r^{4}}}\right]^{1 / 2}
\end{aligned}
$$

(iv) $-\infty<\alpha<1$ case :

In this case, using Eq. A.7) we get,

$$
e^{2 \psi(\bar{r})}=1-\frac{2 M}{\bar{r}}-\frac{\alpha \bar{r}^{2}}{6 \kappa(1-\alpha)}\left[\sqrt{1+\frac{4 \kappa q^{2}(1-\alpha)}{\alpha \bar{r}^{4}}}-1\right]+\frac{4 q^{2}}{3 \bar{r}^{2}}{ }_{2} F_{1}\left(\frac{1}{4}, \frac{1}{2} ; \frac{5}{4} ;-\frac{4 \kappa q^{2}(1-\alpha)}{\alpha \bar{r}^{4}}\right)
$$


where, ${ }_{2} F_{1}(a, b ; c ; z)$ is the hypergeometric function with usual meaning. Here, for $0<\alpha<1$, the $r^{2}\left(\bar{r}_{0}\right)=0$, for $\bar{r}_{0}=\left(\alpha \kappa q^{2}\right)^{1 / 4}$. But, for $\alpha<0$ (i.e. for $\left.\kappa<0\right)$, there is a minimum value of the radius-square, which is $r^{2}\left(\bar{r}_{0}\right)=\left(\frac{\kappa q^{2} \alpha}{1-\alpha}\right)^{1 / 2}$, for $\bar{r}_{0}=0$. Therefore, for $\alpha<0$ or $\kappa<0$, the charge $(q)$ and the mass $(M)$ are distributed over a 2-sphere, but, for $1>\alpha>0$, it is a point charge. This solution also reduces to the Reissner-Nordström solution for very small $\kappa(\kappa \rightarrow \pm 0)$. Here also, the spacetime is singular at the location of the charge and it may be a black hole or naked singularity depending upon the parameter values. The metric functions for this class of solutions are plotted in the Schwarzschild coordinates [eq. (20)] for some parameter values and these are shown in Fig. 2 and Fig. 3 ,

\section{B. Deviation from the solution with Maxwell field}

For $\kappa>0$, the solution with the Maxwell field $(\alpha \rightarrow \infty)$ is given by (using Eqs. [17]18]19]20),

$$
\begin{gathered}
d s^{2}=-\frac{r^{4} h_{1}(r)}{\left(r^{4}-\kappa q^{2}\right)} d t^{2}+\frac{\left(r^{4}-\kappa q^{2}\right)}{\left(r^{4}+\kappa q^{2}\right) h_{1}(r)} d r^{2}+r^{2}\left(d \theta^{2}+\sin ^{2} \theta d \phi^{2}\right) \\
\text { where, } h_{1}(r)=1-\frac{q^{2}}{3 r^{2}}-\frac{2 M r}{\sqrt{r^{4}+\kappa q^{2}}}+\frac{r\left(4 q^{2}\right)^{3 / 4}}{3 \kappa^{1 / 4} \sqrt{r^{4}+\kappa q^{2}}} F\left(\arcsin \left(\frac{\left(4 \kappa q^{2} r^{4}\right)^{1 / 4}}{\sqrt{r^{4}+\kappa q^{2}}}\right) \mid-1\right) .
\end{gathered}
$$

Since, the charge is distributed over a 2-sphere of radius $r_{0}=\left(\kappa q^{2}\right)^{1 / 4}$, therefore $r \geq\left(\kappa q^{2}\right)^{1 / 4}$. For $\kappa<0$, the solution with the Maxwell field $(\alpha \rightarrow-\infty)$ is given by the Eq. (22), but with the replacement of $h_{1}(r)$ by $h_{2}(r)$, where

$$
h_{2}(r)=1-\frac{q^{2}}{3 r^{2}}-\frac{2 M r}{\sqrt{r^{4}+\kappa q^{2}}}+\frac{4 q^{2} r^{2}}{3\left(r^{4}+\kappa q^{2}\right)}{ }_{2} F_{1}\left(\frac{1}{4}, \frac{1}{2} ; \frac{5}{4} ; \frac{4 \kappa q^{2} r^{4}}{\left(r^{4}+\kappa q^{2}\right)^{2}}\right)
$$

The deviation of the EiBI solutions with Born-Infeld electric field from that with the Maxwell field are demonstrated in Fig. 1 and Fig. 2 for $\kappa$ positive and negative respectively. In each figure, $\kappa$-value is fixed, but $\alpha=4 \kappa b^{2}$ takes different values for different solid lines. Therefore, Born-Infeld electric field parameter $\left(b^{2}\right)$ is varied without disturbing the gravity sector. All plots show the variation of metric functions in the Schwarzschild coordinates with physical radial distance $r$. The plots are obtained using Eqs. [17, 18, 19,21, 20, 30, 22, 23, 24. For a given value of $\alpha$, an appropriate aforementioned equation has been used. In some plots $r$ starts with a nonzero minimum value which is due to the charges distributed over a 2 -sphere of finite radius.

In Fig. 1(a), we clearly see that the metric function $\left(g_{t t}\right)$ for different values of $\alpha=4 \kappa b^{2}$ deviate from the case with a Maxwell field (black dashed line) at small $r$. However, at large $r$, plots for different $\alpha$ approach the black dashed line (Maxwell) coinciding with it asymptotically. We also see that, for larger values of $\alpha$ or $b^{2}$, the amount of deviation reduces. In Fig. 1(a) and Fig. 1(b), we note that for the chosen parameter values $(\kappa=1.0, q=0.5, M=1.0)$ the deviation in the horizon radius from the case with a Maxwell field is negligible. We note a clear deviation ( Fig. 1(c) and Fig. 1(d) in the horizon radius for a different value of $q=0.9$. Here, the horizon radius increases with decreasing $\alpha$, or $b^{2}$. In Fig. 1, one can see that for $\alpha=0.5$ and $\alpha=1.5$ the metric functions (both $g_{t t}$ and $g_{r r}$ ) begin from $r=0$ indicating a point charge. In contrast, for $\alpha=3.0$ and the Maxwell limit (black dashed line), the metric functions are limited to a minimum radius of the 2 -sphere where the charge is distributed. From the Fig. 22 one can also note the existence of a minimum radial distance for all $\alpha$ with $\kappa$ negative.

\section{Deviation from the Geonic blackhole solution}

Additionally, we note that, in the limits- $\alpha \rightarrow \pm 0$ and $\kappa \rightarrow \pm 0$, but for a finite $b^{2}$ we get back the geonic black hole solution. The geonic blackhole is a solution in GR coupled to a Born-Infeld electric field due to a point charge [44]. In a geonic black hole scenario, a distant observer associates a total mass which comprises $M$ (the black hole mass) and a pure electromagnetic mass stored as the self energy in the electromagnetic field. If $M$ is zero, the spacetime becomes regular everywhere.

The line element for a geonic blackhole is given as (from Eq. 211),

$$
\begin{aligned}
d s^{2} & =-g_{e}(r) d t^{2}+\frac{d r^{2}}{g_{e}(r)}+r^{2}\left(d \theta^{2}+\sin ^{2} \theta d \phi^{2}\right) \\
\text { where, } g_{e}(r) & =1-\frac{2 M}{r}-\frac{2 q^{2}}{3\left(\sqrt{r^{4}+q^{2} / b^{2}}+r^{2}\right)}+\frac{4 q^{2}}{3 r^{2}}{ }_{2} F_{1}\left(\frac{1}{4}, \frac{1}{2} ; \frac{5}{4} ;-\frac{q^{2}}{b^{2} r^{4}}\right)
\end{aligned}
$$



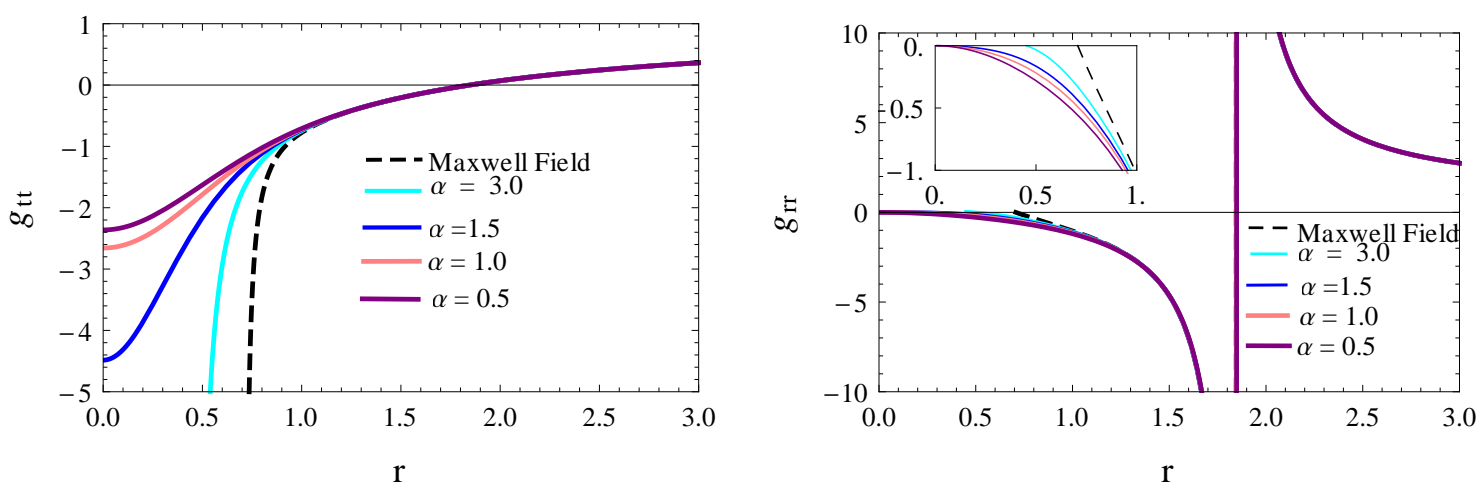

(a) $\kappa=1.0, q=0.5, M=1.0$

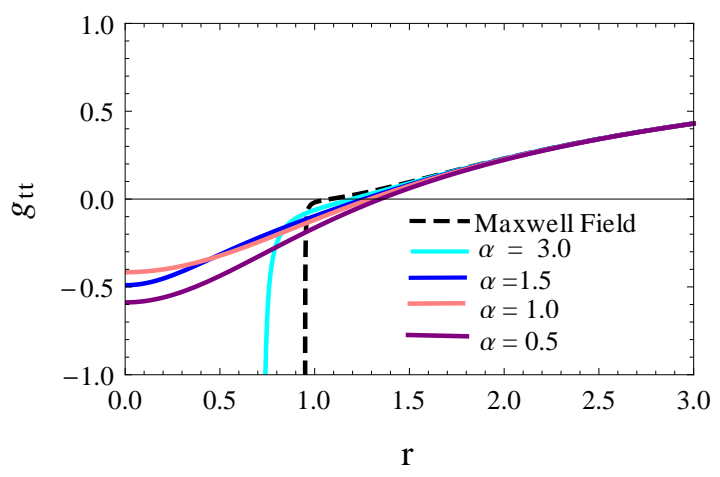

(c) $\kappa=1.0, q=0.9, M=1.0$

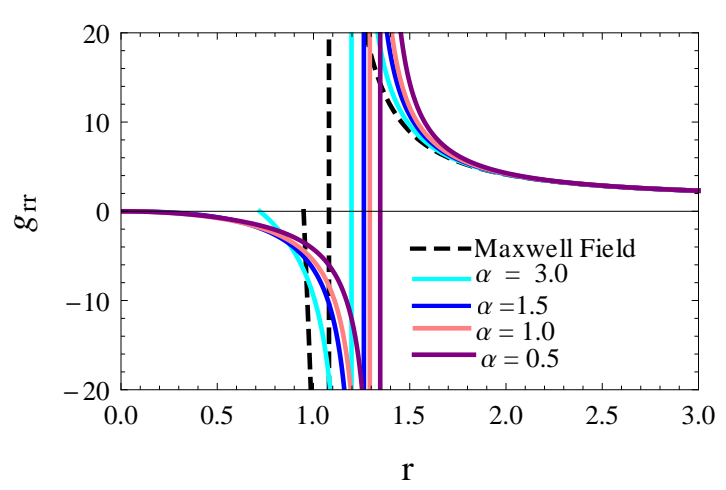

(d) $\kappa=1.0, q=0.9, M=1.0$

FIG. 1. Metric functions in Schwarzschild coordinates are plotted and compared with the EiBI solution for the Maxwell electric field (black dashed line). $g_{t t}$ and $g_{r r}$ are the functions given in the equation (20). In the plots, $\kappa=1.0$, while $\alpha=4 \kappa b^{2}$ takes different values, which means the solutions are with different values of control parameter $\left(b^{2}\right)$ for the nonlinear Born-Infeld electric field.

where, $r$ is again the physical radial distance. Here, $r$ can take any positive value from zero to infinity. The deviation of the EiBI solutions (with different $\kappa$ ) from the geonic blackhole solution (the GR limit) is demonstrated in Fig. 3 with a fixed value of the Born-Infeld electric field parameter $\left(b^{2}\right)$. Here, the gravity sector is varied by changing the value of $\kappa$ without disturbing the matter sector.

The deviation in the metric functions from the geonic blackhole solution (brown dashed line, $\kappa=0$ ) is prominent for different values of $\kappa$ in Fig. 3. However, at larger $r$, all plots approach the brown dashed line. For a larger magnitude of $\kappa$, the deviation is more. In Fig. 3(a) and Fig. 3(b) we see that for the given parameter values, the double horizon exists in the case of the geonic blackhole, though it is absent for other solutions with different values of $\kappa$. From Fig. 3(b), 3(d) and $3(\mathrm{f})$ we note a generic feature that for $\kappa$ positive the horizon radius decreases with increase in magnitude of $\kappa$. But, it is the opposite for negative $\kappa$.

\section{THE $\alpha=1$ CASE}

\section{A. The line element}

For $\alpha=1$, the quadratic equation [Eq. [10)] becomes linear in $V$. Hence, the metric functions $V, U$ and $e^{2 \psi}$ [Eqs. (17), (18), (16)] become

$$
V=1-\frac{\kappa q^{2}}{\bar{r}^{4}}, \quad U=1+\frac{\kappa q^{2}}{\bar{r}^{4}}, \quad \text { and } \quad e^{2 \psi}=1-\frac{2 M}{\bar{r}}+\frac{q^{2}}{\bar{r}^{2}}
$$



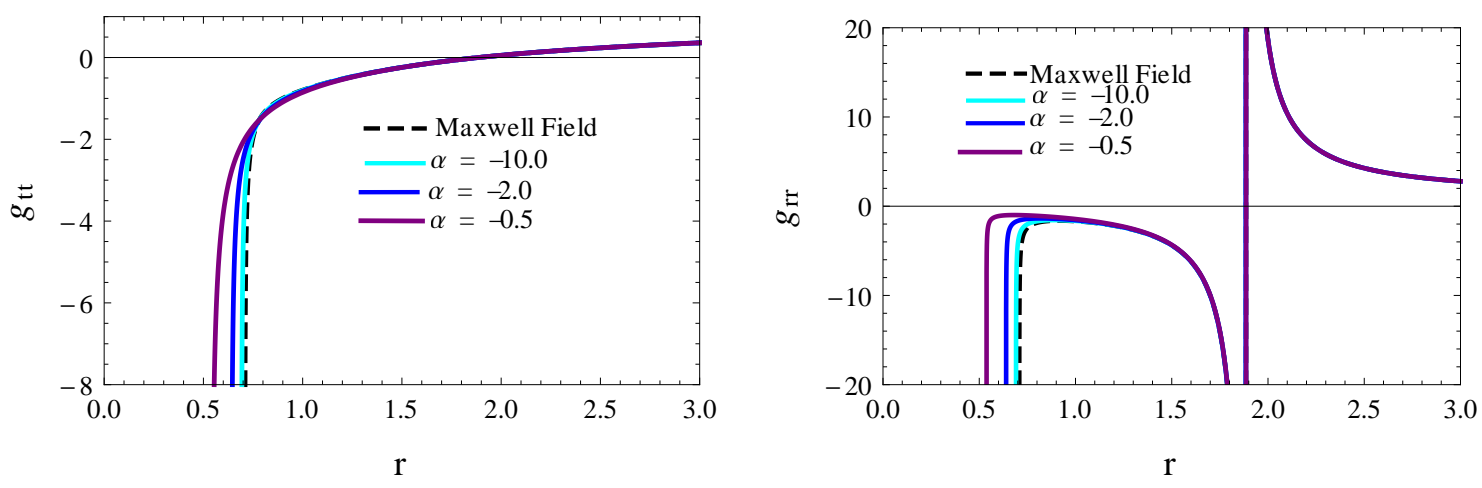

(a) $\kappa=-1.0, q=0.5, M=1.0$

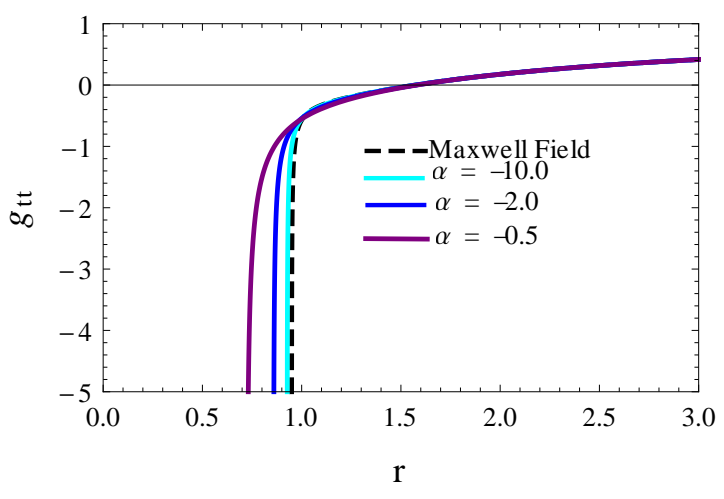

(c) $\kappa=-1.0, q=0.9, M=1.0$

(b) $\kappa=-1.0, q=0.5, M=1.0$

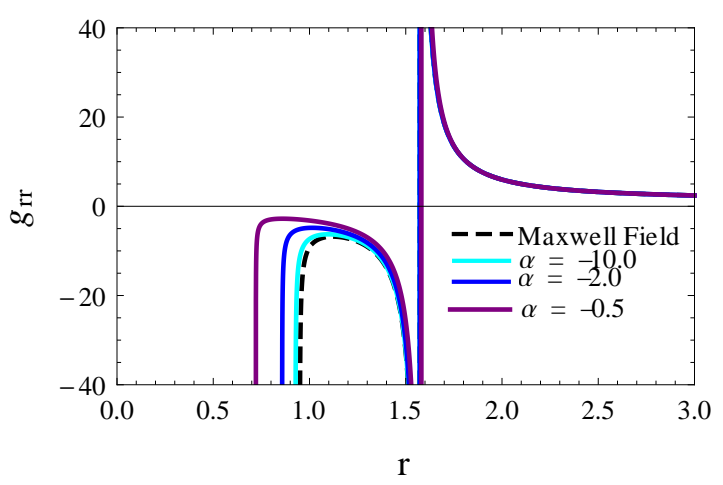

(d) $\kappa=-1.0, q=0.9, M=1.0$

FIG. 2. Metric functions in Schwarzschild coordinates are plotted and compared with the EiBI solution for the Maxwell electric field (black dashed line). $g_{t t}$ and $g_{r r}$ are the functions given in the equation (20). Here, in these plots also, $\kappa$-value is fixed but negative and consequently $\alpha$ is also negative.

Then, the physical line element becomes,

$$
d s^{2}=-\left(1+\frac{\kappa q^{2}}{\bar{r}^{4}}\right)\left(1-\frac{2 M}{\bar{r}}+\frac{q^{2}}{\bar{r}^{2}}\right) d t^{2}+\frac{\left(1+\frac{\kappa q^{2}}{\bar{r}^{4}}\right)}{\left(1-\frac{2 M}{\bar{r}}+\frac{q^{2}}{\bar{r}^{2}}\right)} d \bar{r}^{2}+\left(1-\frac{\kappa q^{2}}{\bar{r}^{4}}\right) \bar{r}^{2}\left(d \theta^{2}+\sin ^{2} \theta d \phi^{2}\right)
$$

Here, the radius-square: $r^{2}(\bar{r})=\bar{r}^{2}-\frac{\kappa q^{2}}{\bar{r}^{2}}$, becomes zero at $\bar{r}=\left(\kappa q^{2}\right)^{1 / 4}$. So, this describes a point charge $q$ with the total mass $M$. This line element [Eq. (28)] clearly shows that, for $\kappa=0$, it becomes the Reissner-Nordström solution. The auxiliary line element is also the same as Reissner-Nordström.

The source for such a spacetime has the following form of the stress-energy tensor in the framework of EiBI gravity:

$$
T_{\nu}^{\mu}=\operatorname{diag} \cdot\left\{-\frac{q^{2}}{8 \pi\left(\bar{r}^{4}-\kappa q^{2}\right)},-\frac{q^{2}}{8 \pi\left(\bar{r}^{4}-\kappa q^{2}\right)}, \frac{q^{2}}{8 \pi\left(\bar{r}^{4}+\kappa q^{2}\right)}, \frac{q^{2}}{8 \pi\left(\bar{r}^{4}+\kappa q^{2}\right)}\right\}
$$

One may verify that the stress energy satisfies the weak/null energy conditions [54].

In the Schwarzschild gauge, the line element takes the form

$$
d s^{2}=-f(r) d t^{2}+\frac{2 r^{2}}{\left(r^{2}+\sqrt{r^{4}+4 \kappa q^{2}}\right) f(r)} d r^{2}+r^{2}\left(d \theta^{2}+\sin ^{2} \theta d \phi^{2}\right)
$$

where, the redshift function, $f(r)=\left(1+\frac{4 \kappa q^{2}}{\left(r^{2}+\sqrt{r^{4}+4 \kappa q^{2}}\right)^{2}}\right)\left[1-\frac{2 \sqrt{2} M}{\sqrt{r^{2}+\sqrt{r^{4}+4 \kappa q^{2}}}}+\frac{2 q^{2}}{r^{2}+\sqrt{r^{4}+4 \kappa q^{2}}}\right]$. At the horizon- 

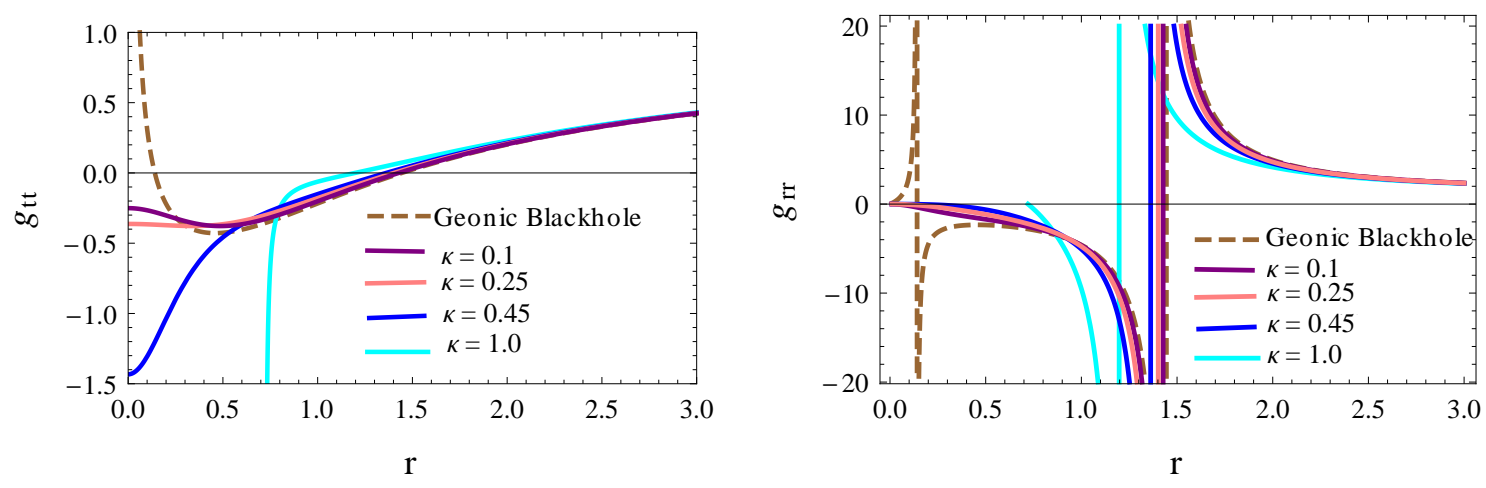

(a) $b^{2}=1.0, q=0.9, M=1.0$
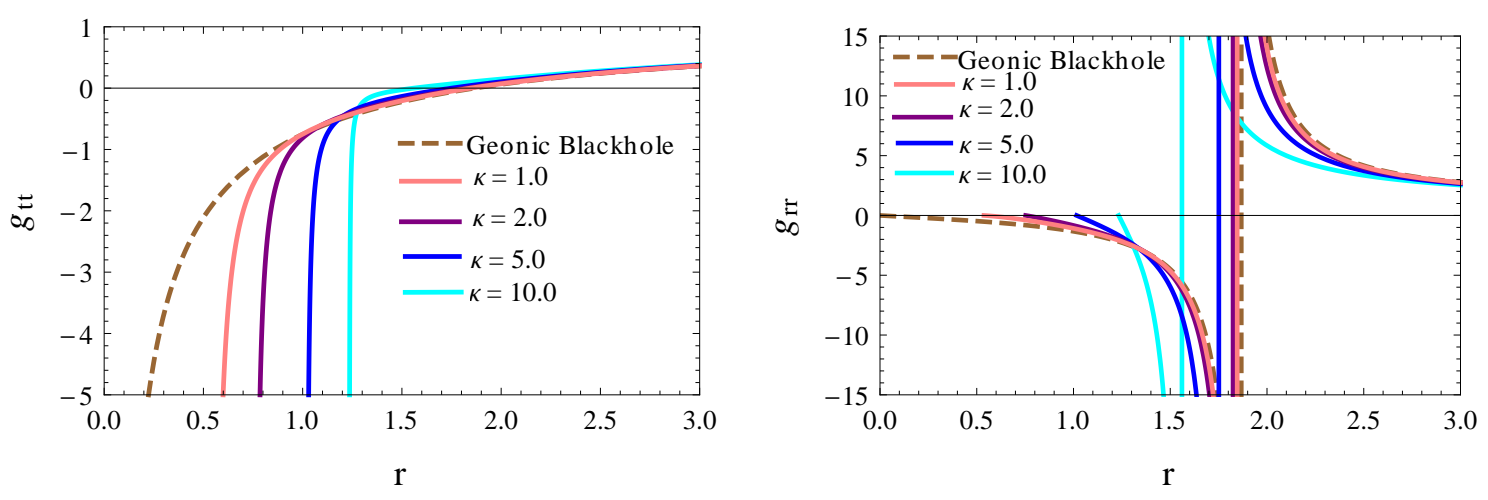

(c) $b^{2}=1.0, q=0.5, M=1.0$

(d) $b^{2}=1.0, q=0.5, M=1.0$
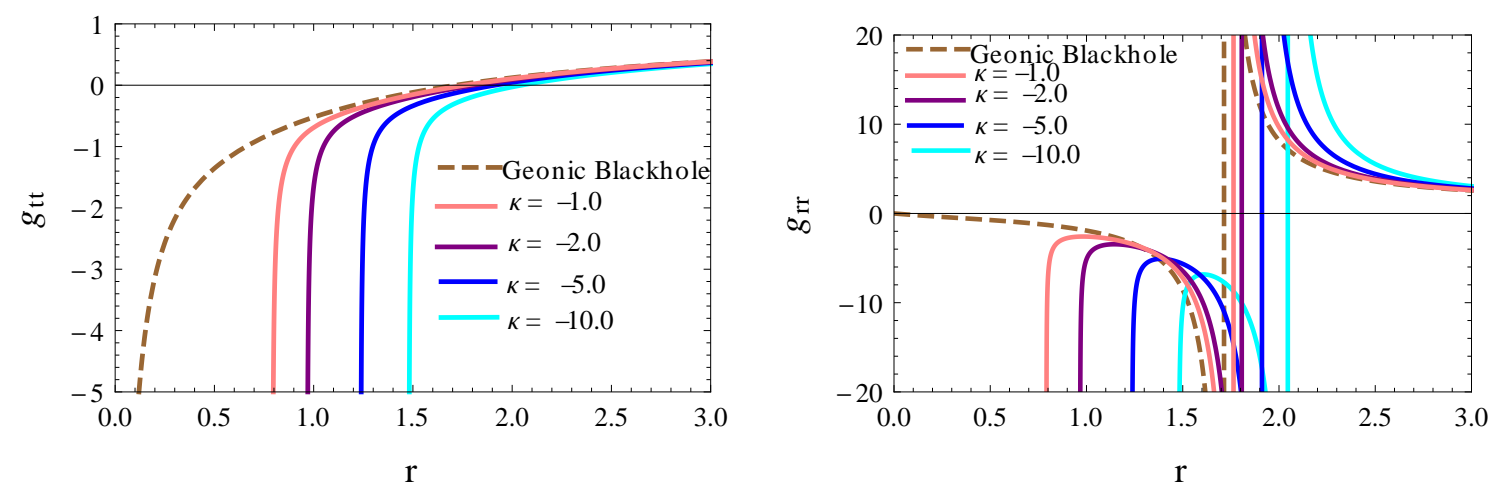

(e) $b^{2}=1.0, q=0.7, M=1.0$

(f) $b^{2}=1.0, q=0.7, M=1.0$

FIG. 3. Metric functions in Schwarzschild coordinates are plotted and compared with the geonic blackhole solution (brown dashed line). $g_{t t}$ and $g_{r r}$ are the functions given in the equation (20). Here, in these plots, the Born-Infeld electric field parameter $\left(b^{2}\right)$ has the fixed value $b^{2}=1$, but EiBI gravity parameter $\kappa$ is varied.

radius, $r_{h z}, f\left(r_{h z}\right)=0$. The expression for the horizon-radius is therefore given by,

$$
r_{h z}^{2}=\frac{\left(M \pm \sqrt{M^{2}-q^{2}}\right)^{4}-\kappa q^{2}}{\left(M \pm \sqrt{M^{2}-q^{2}}\right)^{2}}
$$

Here, we note that a horizon does not exist for $q>M$, similar to the Reissner-Nordström case. For $q=M$, we have $r_{h z}^{2}=q^{2}-\kappa$. So, unlike the Reissner-Nordström extremal solution, for each value of $q=M$, there is an upper limit on $\kappa$ for the existence of the horizon, (see the left panel of the Fig. 4). From the right panel of the Fig. (4) we note that, for $q<M$, for sufficiently small values of $\kappa$ there may be double horizon. For a range of higher values of $\kappa$ a single-horizon exists, and for even more high values of $\kappa$ the horizon vanishes. However, in the Reissner-Nordström 
counterpart, we always have the double horizon.

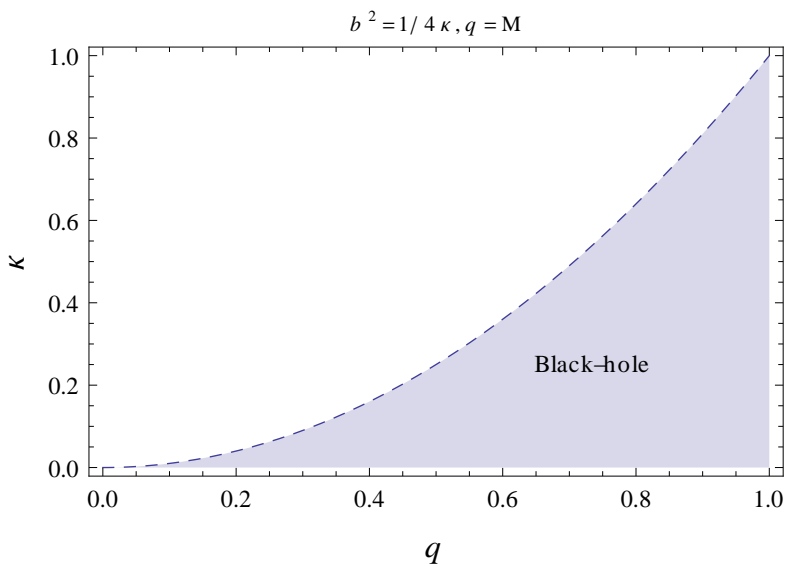

(a)

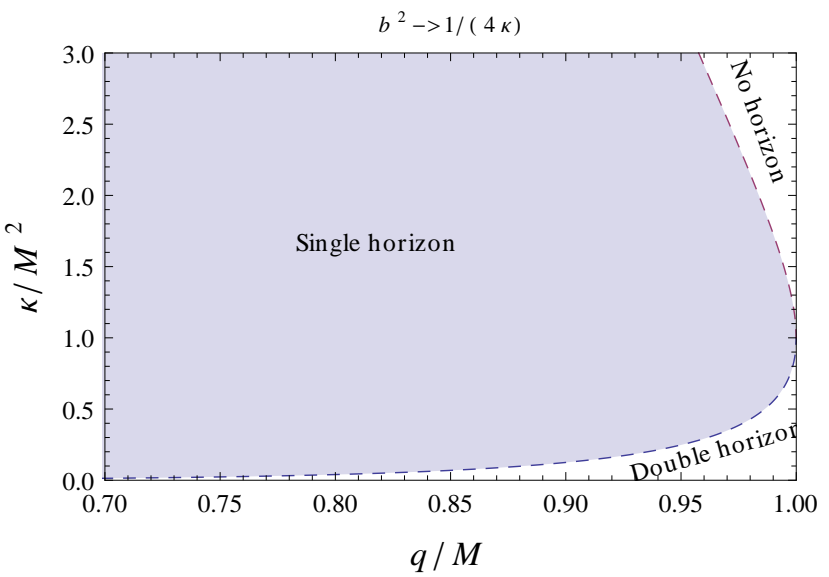

(b)

FIG. 4. (Left panel)the parameter space: $q^{2}-\kappa$ showing the existence/non-existence of horizon, for $q=M$. (Right panel) the same plot for $q \neq M$ case.

One can verify that, in general, all the scalar quantities, like the Ricci scalar $\left(\mathcal{R}=g^{i j} R_{i j}\right), R_{i j} R^{i j}$, and the Kretschmann scalar $\left(R_{i j k l} R^{i j k l}\right)$, diverge at the location of the point charge, i.e. at $\bar{r}=\left(\kappa q^{2}\right)^{1 / 4}$, or, $r=0$. However, for a special choice, $q=\sqrt{\kappa} / 3$ and $M=2 \sqrt{\kappa} / 3 \sqrt{3}$, the Ricci scalar becomes regular everywhere. But, the spacetime still remains singular at the location of the point charge, for such a choice, since the Kretschmann scalar and $R_{i j} R^{i j}$ diverge. Fig. 5 demonstrates the singularity for two different value of the parameters. So, the spacetime is eventually singular, but may or may not be covered by a horizon. However, we show that the strength of the electric field at the location of the charge remains finite. For this, we consider a static observer having the four velocity, $u^{\mu}=$ $\left\{e^{-\psi} / \sqrt{U}, 0,0,0\right\}$. The electric field vector is defined as $E_{\mu}=u^{\nu} F_{\mu \nu}$. So, the square of the strength of the electric field is $E^{2}=E^{\mu} E_{\mu}=\frac{q^{2}}{V^{2} \bar{r}^{4}+q^{2} / b^{2}}$. At the location of the charge, $E^{2}=\frac{q^{2}}{r^{4}\left(\bar{r}_{0}\right)+q^{2} / b^{2}}=b^{2}=1 / 4 \kappa$ (in this case, $\left.r\left(\bar{r}_{0}\right)=0\right)$. The energy density observed by such a static observer is $\rho_{o b s}=u^{\mu} u^{\nu} T_{\mu \nu}=\frac{b^{2}}{4 \pi}\left(\frac{\sqrt{V^{2} \bar{r}^{4}+q^{2} / b^{2}}}{V \bar{r}^{2}}-1\right)$. In this case, $\rho_{o b s}=\frac{q^{2}}{8 \pi\left(\bar{r}^{4}-\kappa q^{2}\right)}$. It diverges at the location of the point charge $\left(\bar{r}=\bar{r}_{0}=\left(\kappa q^{2}\right)^{1 / 4}\right)$. We note that the non-singular feature of the strength of the electric field remains even in the solution of EiBI gravity coupled to Maxwell's electrodynamics [8, 40] and this is due to the Born-Infeld like structure in the gravity sector. However, this does not ensure the nonsingularity of the spacetime. In [8, 40], the charge is not point-like, but distributed over a spherical surface. $\rho_{o b s}$ remains finite, though the scalar invariants diverge at this surface. But, in our solution, both the energy density and the curvature scalar are singular. A non-linear correlation between the gravity and the physical matter sector in the EiBI theory is thus revealed.

\section{B. Null geodesics}

Let us now turn to the null geodesics in this spacetime. On the equatorial plane $(\theta=\pi / 2)$, for null geodesics, we have, from Eq. (28),

$$
\frac{\dot{r}^{2}}{2}\left(1-\frac{\kappa q^{2}}{\bar{r}^{4}}\right)+\frac{L^{2}}{2 r^{2}(\bar{r})}\left(1+\frac{\kappa q^{2}}{\bar{r}^{4}}\right)\left(1-\frac{2 M}{\bar{r}}+\frac{q^{2}}{\bar{r}^{2}}\right)=\frac{E^{2}}{2}
$$

where, $E=\left(1+\frac{\kappa q^{2}}{\bar{r}^{4}}\right)\left(1-\frac{2 M}{\bar{r}}+\frac{q^{2}}{\bar{r}^{2}}\right) \dot{t}, L=r^{2}(\bar{r}) \dot{\phi}$ are two conserved quantities and $\dot{t}, \dot{\phi}, \dot{r}$ are defined as $\dot{t}=\frac{d t}{d \lambda}, \dot{\phi}=$ $\frac{d \phi}{d \lambda}, \dot{r}=\frac{d r}{d \bar{r}} \frac{d \bar{r}}{d \lambda}, \lambda$ is the affine parameter. So, from Eq. (32) we identify the effective potential for the null geodesics as

$$
V_{\text {eff }}(r)=\frac{L^{2}}{2 r^{2}}\left(1+\frac{\kappa q^{2}}{\bar{r}^{4}}\right)\left(1-\frac{2 M}{\bar{r}}+\frac{q^{2}}{\bar{r}^{2}}\right)
$$

where, $\bar{r}^{2}=\left(r^{2}+\sqrt{r^{4}+4 \kappa q^{2}}\right) / 2$. The aforementioned effective potential is useful to study the propagation of gravitational signals in the current scenario. We discuss photon propagation in the following subsection. 


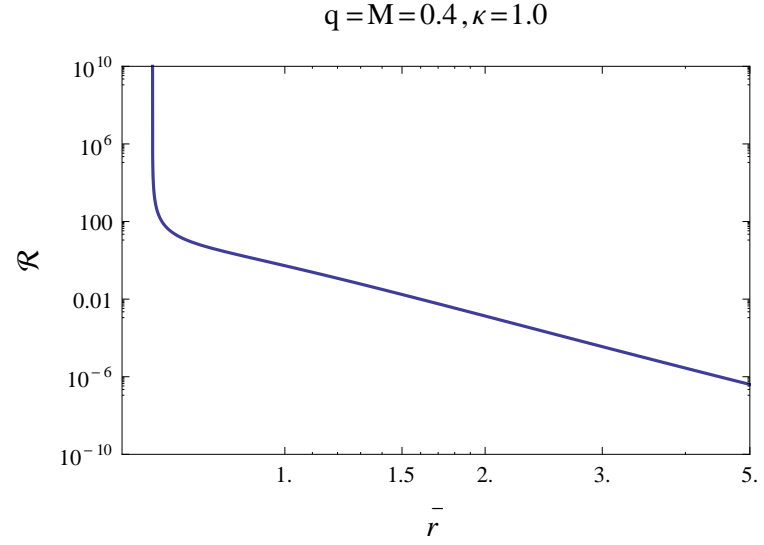

(a)

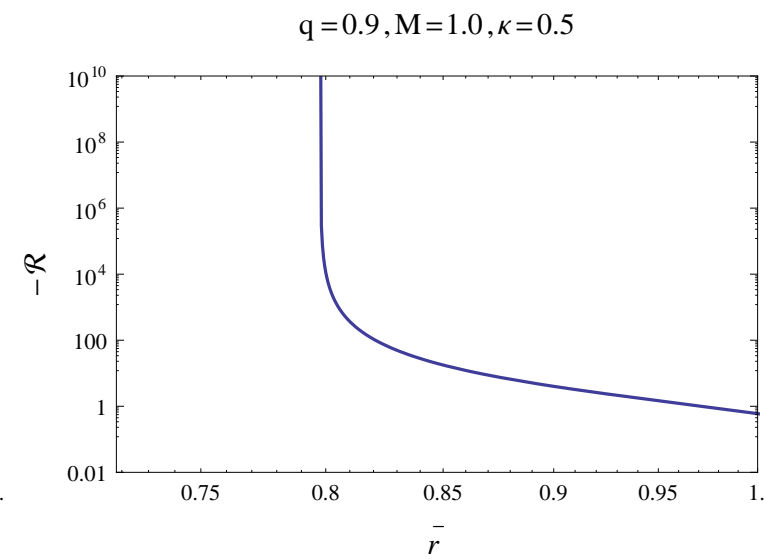

(b)

FIG. 5. (Left panel)the ricci scalar $(\mathcal{R})$ is plotted as a function of $\bar{r}$ for the parameter values, $q=M=0.4, \kappa=1.0$. The plot shows that $\mathcal{R}$ diverges at the location of the charge, i.e. $\bar{r}=\left(\kappa q^{2}\right)^{1 / 4}$ or, $r=0$. (Right panel) the same plot for a $q \neq M$ case. Here, $\mathcal{R}$ diverges to negative values.

\section{The propagation of photons}

Photons (lightlike particles) in Born-Infeld electromagnetism (BI photon), no longer propagate along the null geodesics of the background spacetime geometry. This is generally true for nonlinear electrodynamics [55]. Instead, the BI photon trajectories are determined by the null geodesics of the so-called effective geometry, given by [55, [56],

$$
g_{\text {eff }}^{\mu \nu}=\left(1+\frac{1}{b^{2}} F\right) g^{\mu \nu}+\frac{1}{b^{2}} F_{\sigma}^{\mu} F^{\sigma \nu}
$$

where, $F=\frac{1}{2} F_{\alpha \beta} F^{\alpha \beta}$ and $g_{\mu \nu}$ defines the metric of the background spacetime. Such an effective geometry is induced due to nonlinear self-interaction of the photon. Here, in Eq. (34), we assume the same $F_{\mu \nu}$ which generates background spacetime geometry. Then, the effective line element becomes

$$
d s_{\text {eff }}^{2}=-\left(1+\frac{\kappa q^{2}}{\bar{r}^{4}}\right)\left(1-\frac{2 M}{\bar{r}}+\frac{q^{2}}{\bar{r}^{2}}\right) d t^{2}+\frac{\left(1+\frac{\kappa q^{2}}{\bar{r}^{4}}\right)}{\left(1-\frac{2 M}{\bar{r}}+\frac{q^{2}}{\bar{r}^{2}}\right)} d \bar{r}^{2}+\frac{\left(1+\frac{\kappa q^{2}}{\bar{r}^{4}}\right)^{2}}{\left(1-\frac{\kappa q^{2}}{\bar{r}^{4}}\right)} \bar{r}^{2}\left(d \theta^{2}+\sin ^{2} \theta d \phi^{2}\right)
$$

Since our interest is to study the null geodesics of the effective geometry, we take out the conformal factor $\left(1+\frac{\kappa q^{2}}{\bar{r}^{4}}\right)$ and rewrite the effective line element as,

$$
d s_{e f f}^{2}=-\left(1-\frac{2 M}{\bar{r}}+\frac{q^{2}}{\bar{r}^{2}}\right) d t^{2}+\frac{d \bar{r}^{2}}{\left(1-\frac{2 M}{\bar{r}}+\frac{q^{2}}{\bar{r}^{2}}\right)}+\frac{\left(1+\frac{\kappa q^{2}}{\bar{r}^{4}}\right)}{\left(1-\frac{\kappa q^{2}}{\bar{r}^{4}}\right)} \bar{r}^{2}\left(d \theta^{2}+\sin ^{2} \theta d \phi^{2}\right)
$$

The effective geometry becomes exactly the same as the Reissner-Nordström solution when $\kappa=0$, which is expected. The analysis of the squared area radius in the effective geometry, $\left[r^{2}=\bar{r}^{2}\left(\frac{\bar{r}^{4}+\kappa q^{2}}{\bar{r}^{4}-\kappa q^{2}}\right)\right]$, shows that the area radius, or, the physical radial distance $r$ becomes infinity both at $\bar{r}=\bar{r}_{0}=\left(\kappa q^{2}\right)^{1 / 4}$ and $\bar{r} \rightarrow \infty$, but has a minimum $r_{t h}^{2}=\frac{\sqrt{\kappa} q}{(\sqrt{5}-2)^{1 / 2}}\left(\frac{\sqrt{5}-1}{3-\sqrt{5}}\right)$ for $\bar{r}_{t h}=\left(\frac{\kappa q^{2}}{\sqrt{5}-2}\right)^{1 / 4}$ (similar to the throat radius square of a wormhole). This implies that for each physical radial distance $r \in\left[r_{t h}, \infty\right]$, there exists a $\bar{r} \in\left[\bar{r}_{t h}, \infty\right]$ and a $\bar{r} \in\left[\bar{r}_{0}, \bar{r}_{t h}\right]$. But these two sets of ranges of $\bar{r}$ describe two different geometries. We are interested in studying the propagation of photons coming from and going towards asymptotically flat regions of the given spacetime. Hence, we focus on $\bar{r}_{t h} \leq \bar{r}<\infty$, which describes an asymptotically flat geometry.

Now, for the null geodesics, we have

$$
\frac{1}{2} \dot{\bar{r}}^{2}+\frac{L^{2}}{2 \bar{r}^{2}}\left(1-\frac{2 M}{\bar{r}}+\frac{q^{2}}{\bar{r}^{2}}\right)\left(\frac{\bar{r}^{4}-\kappa q^{2}}{\bar{r}^{4}+\kappa q^{2}}\right)=\frac{E^{2}}{2}
$$


where, $E=\left(1-\frac{2 M}{\bar{r}}+\frac{q^{2}}{\bar{r}^{2}}\right) \dot{t}$ and $L=r^{2}(\bar{r}) \dot{\phi}$ are two conserved quantities. One can also rewrite the Eq. (37) in terms of physical radial distance $r$ using the relation $r^{2}(\bar{r})=\bar{r}^{2}\left(\frac{\bar{r}^{4}+\kappa q^{2}}{\bar{r}^{4}-\kappa q^{2}}\right)$. So, the effective potential for the BI photons become

$$
V_{e f f}(\bar{r})=\frac{L^{2}}{2 \bar{r}^{2}}\left(1-\frac{2 M}{\bar{r}}+\frac{q^{2}}{\bar{r}^{2}}\right)\left(\frac{\bar{r}^{4}-\kappa q^{2}}{\bar{r}^{4}+\kappa q^{2}}\right)
$$

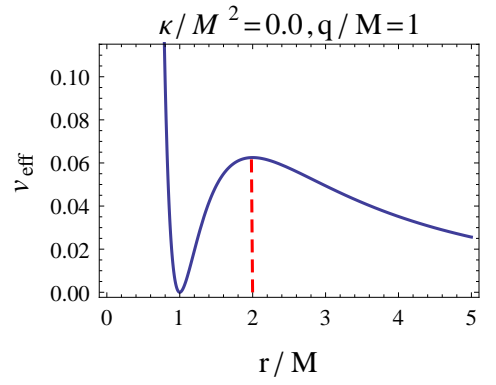

(a)

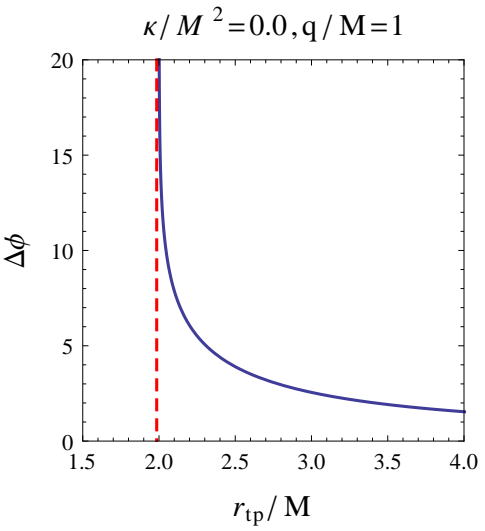

(d)

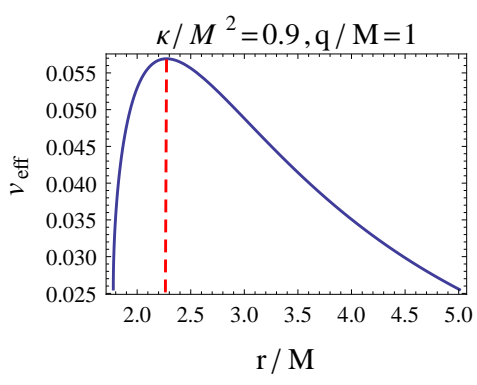

(b)

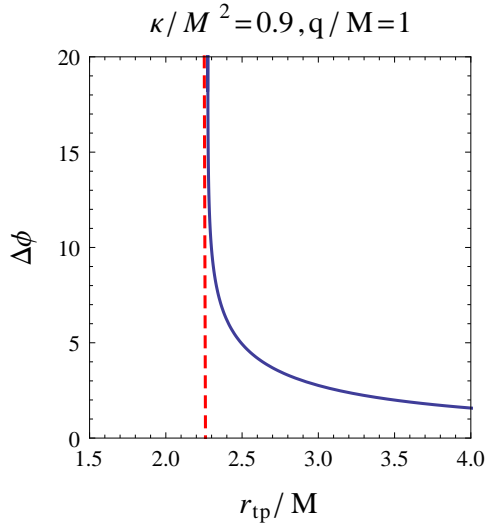

(e)

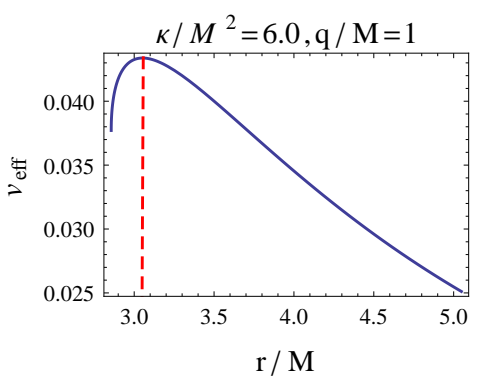

(c)

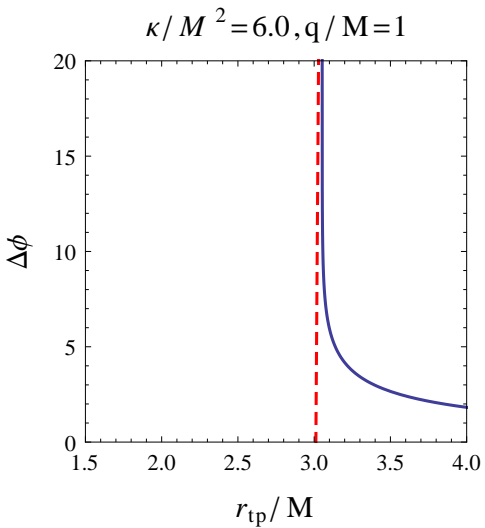

(f)

FIG. 6. (Top panel)The effective potential [Eq. (38)] is plotted as a function of the dimensionless quantity $r / M$, where $r(\bar{r})$ is the radius of the 2 -sphere labelled by the radial coordinate $\bar{r}$ and $M$ is the mass. In the parametric plot, the $(\bar{r})$ values are in the range $\bar{r}_{t h} \leq \bar{r}<\infty$. In the plot, $v_{e f f}=2 M^{2} V_{e f f} / L^{2}$. The parameter values are specified on the top of the figures. The vertical red dashed line in each plot shows the correspondence between the photon sphere radius and the local maxima of the effective potential. (Bottom panel)The exact deflection angle $(\Delta \phi)$ is plotted as a function of $r_{t p} / M$, where $r_{t p}$ is the physical radial distance at the turning point. In each figure, $\Delta \phi$ rises sharply along the vertical red dashed line. The corresponding $r_{t p} / M$ relates to the photon sphere radius.

We plot the effective potential for different values of $\kappa / M^{2}$ (a dimensionless parameter) and extract the essential features. A few plots are shown in the top panel of the Fig. 6. In these plots, the effective potential is rescaled as $v_{e f f}=2 M^{2} V_{e f f} / L^{2}$ and is plotted as a function of the dimensionless quantity $r / M$. In these parametric plots, the parameter $(\bar{r})$ value ranges from $\bar{r}_{t h}$ to infinity. The maxima of the effective potential correspond to the unstable circular orbits for photons. The corresponding spherical surface is called the 'photon sphere' [54, 57, 58]. In the Reissner-Nordström solution, the radius of the photon sphere depends on $q$ and $M$. In our solution, we note that, it depends additionally on $\kappa$. As an example, we consider the case for $q / M=1$. In the Reissner-Nordström solution, the radius of this photon sphere is $r_{p s}=2 M=2 q$ (see Fig. 6(a)). In our solution, we have a different picture. Here, from the figures of the top panel of Fig. 6. we see that as $\kappa$ is increased the radius of the photon sphere increases. This feature becomes more clear when we look at the plot of deflection angles $(\Delta \phi)$ in the bottom panel of Fig. 6. Near photon sphere, the deflection angle becomes infinitely large, indicating multiple full rounds of light rays around the lensing object. Consequently, a sequence of a large number of highly demagnified relativistic images may arise [59, 60].

Analysing the plots of the effective potential for greater values of $\kappa / M^{2}$, one can easily verify that the radius of the photon sphere $\left(r_{p s}\right)$ increases but becomes closer and closer to the throat radius $\left(r_{t h}\right)$ as $\kappa / M^{2}$-value increases. 
Strong deflection: In this effective geometry, the exact expression for the deflection angle [54] becomes,

$$
\begin{aligned}
\Delta \phi= & 2\left|\phi(\infty)-\phi\left(r_{t p}\right)\right|-\pi \\
= & 2 \int_{\bar{r}_{t p}}^{\infty} \frac{\left(\bar{r}^{4}-\kappa q^{2}\right)}{\bar{r}^{2}\left(\bar{r}^{4}+\kappa q^{2}\right)}\left[\frac{1}{\bar{r}_{t p}^{2}}\left(\frac{\bar{r}_{t p}^{4}-\kappa q^{2}}{\bar{r}_{t p}^{4}+\kappa q^{2}}\right)\left(1-\frac{2 M}{\bar{r}_{t p}}+\frac{q^{2}}{\bar{r}_{t p}^{2}}\right)-\frac{1}{\bar{r}^{2}}\left(\frac{\bar{r}^{4}-\kappa q^{2}}{\bar{r}^{4}+\kappa q^{2}}\right)\left(1-\frac{2 M}{\bar{r}}+\frac{q^{2}}{\bar{r}^{2}}\right)\right]^{-1 / 2} d \bar{r} \\
& -\pi
\end{aligned}
$$

where, $r^{2}\left(\bar{r}_{t p}\right)=\bar{r}_{t p}^{2} \frac{\left(\bar{r}_{t p}^{4}+\kappa q^{2}\right)}{\left(\bar{r}_{t p}^{4}-\kappa q^{2}\right)}$. Here, $r_{t p}$ indicates the turning point, i.e. $\left.\frac{d r}{d \phi}\right|_{r_{t p}}=\left.\frac{\dot{r}}{\dot{\phi}}\right|_{r_{t p}}=0$. The deflection angle is plotted as a function of the dimensionless parameter $r_{t p} / M$ and the figures are shown in the bottom panel of Fig. 6. Fig. 6(d) (for $\kappa / M^{2}=0, q / M=1$ ) shows that, in the extremal Reissner-Nordström case, the deflection angle increases rapidly $(>>2 \pi)$ near the photon sphere $\left(r_{t p}=r_{p s}=2 M\right)$. From the next two figures (Fig. 6(e) and Fig. 6(f) , we see that the nature of plots do not change. However, as the $\kappa / M^{2}$ value increases, it is clearly seen that radius of the photon sphere increases from the value $2 M$.

Weak deflection: Let us now look for the leading order contribution of $\kappa$ in the weak-deflection angle formula. We compare this result with the Reissner-Nordström case. We obtain the weak deflection angle perturbatively 61. For a spherically symmetric static line element, $d s^{2}=-A(\bar{r}) d t^{2}+B(\bar{r}) d \bar{r}^{2}+\bar{r}^{2} C(\bar{r}) d \Omega^{2}$, the radial geodesic equation is

$$
2 B \ddot{\vec{r}}+B^{\prime} \dot{r}^{2}+A^{\prime} \dot{t}^{2}-\left(\bar{r}^{2} C\right)^{\prime} \dot{\phi}^{2}=0
$$

With the conserved quantities, $E=A \dot{t}, L=C \bar{r}^{2} \dot{\phi}$, and the new variable $u=\frac{1}{\bar{r}}$, for the null geodesics, the Eq. (40) becomes 61]

$$
\frac{d^{2} u}{d \phi^{2}}+\frac{C}{B} u=-\frac{1}{2} u^{2} \frac{d}{d u}\left(\frac{C}{B}\right)+\frac{1}{2 b_{0}^{2}} \frac{d}{d u}\left(\frac{C^{2}}{A B}\right)
$$

where, $b_{0}=L / E$ is the impact parameter. In the Reissner-Nordström case, $\left(A=B^{-1}=1-2 M u+q^{2} u^{2}, C=1\right)$, the Eq. (41) becomes,

$$
\frac{d^{2} u}{d \phi^{2}}+u=3 M u^{2}-2 q^{2} u^{3}
$$

In our case, $A=B^{-1}=\left(1-2 M u+q^{2} u^{2}\right)$, and $C=\left(1+\kappa q^{2} u^{4}\right) /\left(1-\kappa q^{2} u^{4}\right)$. We assume small $\kappa\left(\kappa q^{2} u^{4}<<1\right)$ and write the Eq. (41) keeping only the first order terms in $\kappa$,

$$
\frac{d^{2} u}{d \phi^{2}}+u=3 M u^{2}-\left(2-\frac{8 \kappa}{b_{0}^{2}}\right) q^{2} u^{3}-6 \kappa q^{2} u^{5}+14 \kappa q^{2} M u^{6}-8 \kappa q^{4} u^{7}
$$

We set $\epsilon=M u_{N}$, where $u_{N}=1 / \bar{r}_{N}$ and $b_{0}=r_{N}=\bar{r}_{N}\left(\frac{\bar{r}_{N}^{4}+\kappa q^{2}}{\bar{r}_{N}^{4}-\kappa q^{2}}\right)\left(r_{N}\right.$ is the Newtonian distance of closest approach). Further, we define a dimensionless variable $\xi=u / u_{N}$. Thereafter, we write the Eq. (43) to second order in $\epsilon$,

$$
\frac{d^{2} \xi}{d \phi^{2}}+\xi \approx 3 \epsilon \xi^{2}-\epsilon^{2}\left(2-\frac{8 \kappa}{b_{0}^{2}}\right) \frac{q^{2}}{M^{2}} \xi^{3}
$$

Expressing $\xi$ as $\xi=\xi_{0}+\epsilon \xi_{1}+\epsilon^{2} \xi_{2}+\ldots .$. , and substituting them in the Eq. (44) we find the following set of equations, by collecting terms of different order:

$$
\begin{aligned}
& \frac{d^{2} \xi_{0}}{d \phi^{2}}+\xi_{0}=0 \\
& \frac{d^{2} \xi_{1}}{d \phi^{2}}+\xi_{1}-3 \xi_{0}^{2}=0 \\
& \frac{d^{2} \xi_{2}}{d \phi^{2}}+\xi_{2}-6 \xi_{0} \xi_{1}+2\left(1-\frac{4 \kappa}{b_{0}^{2}}\right) \frac{q^{2}}{M^{2}} \xi_{0}^{3}=0
\end{aligned}
$$

Solving the equations (45), (46), and (47) we get the approximate inverse radial distance,

$$
\begin{aligned}
u \simeq & u_{N} \cos \phi+M u_{N}^{2}\left(\frac{3}{2}-\frac{1}{2} \cos (2 \phi)\right)+ \\
& \frac{3 M^{2} u_{N}^{3}}{16}\left(\left(20-4\left(1-\frac{4 \kappa}{b_{0}^{2}}\right) \frac{q^{2}}{M^{2}}\right) \phi \sin \phi+\left(1+\frac{q^{2}}{3 M^{2}}\left(1-\frac{4 \kappa}{b_{0}^{2}}\right)\right) \cos (3 \phi)\right)
\end{aligned}
$$


Assuming, $u \rightarrow 0$ at $\phi=\pi / 2+\delta$, we solve for $\delta$ using the Eq. (48). For a small deflection angle, we find

$$
\delta \approx 2 M u_{N}+\frac{3 \pi M^{2} u_{N}^{2}}{8}\left[5-\left(1-\frac{4 \kappa}{b_{0}^{2}}\right) \frac{q^{2}}{M^{2}}\right]+\mathcal{O}\left(M^{3} u_{N}^{3}\right)
$$

The deflection angle is $\Delta \phi=2 \delta$. To get the first order in $\kappa$ contribution in the deflection angle, we use $b_{0} \approx 1 / u_{N}$. At the distance of closest approach, $\bar{r}_{t p}=1 / u(0)$. Converting from $u_{N}$ to $u(0)$, substituting $u(0)=1 / \bar{r}_{t p}$, with $\bar{r}_{t p} \approx r_{t p}\left(1-\kappa q^{2} / r_{t p}^{4}+\mathcal{O}\left(\kappa^{2}\right)\right)$, we finally obtain the approximate weak deflection angle formula as,

$$
\Delta \phi_{\text {weak }} \simeq \frac{4 M}{r_{t p}}+\frac{M^{2}}{r_{t p}^{2}}\left(\frac{15}{4} \pi-4\right)-\frac{3 \pi}{4}\left(1-\frac{4 \kappa}{r_{t p}^{2}}\right) \frac{q^{2}}{r_{t p}^{2}}
$$

In this formula, the term involving $\kappa$ is the new contribution to weak deflection angle. If $\kappa=0$, the expression becomes the same as in the Reissner-Nordström case 62. By extending the calculation upto the fourth order perturbation (i.e. $\mathcal{O}\left(\epsilon^{4}\right)$ ), one can verify that the sign of the leading order in $\kappa$ term that appears in the fourth order correction $\left(1 / r_{t p}^{4}\right.$-term) in the deflection angle, is still positive. This term involving $\kappa$ increases the net deflection angle. As the value of $\kappa$ is increased, the deflection angle also increases further. Earlier, we have seen that, with the increase of $\kappa$, the radius of photon-sphere increases. Thus, the study of both strong and weak deflection seem to suggest an overall attractive effect of $\kappa$.

\section{EFFECTIVE POTENTIAL AND PHOTON PROPAGATION FOR $\alpha \neq 1$ SOLUTIONS}

In this section we investigate the effective potential, photon sphere, and light propagation for $\alpha \neq 1$ solutions through numerical graph and plots. We also compare these with that for the Maxwell electric field by taking different values of $b^{2}$ (or, $\alpha$ with fixed $\kappa$ ).

The effective metric for the BI photon propagating in the background spacetime which is the EiBI solution with Born-Infeld elecric field $(\alpha \neq 1)$, is derived from Eq. (34) using Eqs. 66 7 as,

$$
d s_{e f f}^{2}=-U(\bar{r}) e^{2 \psi(\bar{r})} d t^{2}+U(\bar{r}) e^{-2 \psi(\bar{r})} d \bar{r}^{2}+\left(\frac{\alpha V^{2}(\bar{r}) \bar{r}^{4}+4 \kappa q^{2}}{\alpha V(\bar{r}) \bar{r}^{2}}\right)\left(d \theta^{2}+\sin ^{2} \theta d \phi^{2}\right)
$$

where, $U(\bar{r}), V(\bar{r})$, and $e^{2 \psi(\bar{r})}$ have the expressions given by the Eqs. 17, 18, and 19] or, 21]. Using Eq. (51), and following the same analysis as described in section IV, the effective potential for BI photon (for $\alpha \neq 1$ ) is derived as,

$$
\begin{aligned}
V_{\text {eff }}(\bar{r}) & =\frac{U(\bar{r}) e^{2 \psi(\bar{r})} L^{2}}{2 r^{2}(\bar{r})} \\
\text { where, } r^{2}(\bar{r}) & =\left(\frac{\alpha V^{2}(\bar{r}) \bar{r}^{4}+4 \kappa q^{2}}{\alpha V(\bar{r}) \bar{r}^{2}}\right) .
\end{aligned}
$$

Here, the effective potential as a function of the physical radial distance, i.e. $V_{\text {eff }}(r)$, is to be determined in a parametric form as given by Eqs. [(52), (53)] .

On the other hand, from Eq. (22), we get the effective potential for the Maxwell photon as

$$
V_{e f f}^{\operatorname{Max}}(r)=\frac{r^{2} h_{1}(r) L^{2}}{2\left(r^{4}-\kappa q^{2}\right)}
$$

where, $h_{1}(r)$ is given by Eq. (23). In Eq. (54), the effective potential is written directly as a function of the physical radial distance $r$. For $\kappa<0, h_{1}(r)$ is replaced by $h_{2}(r)$ (given by Eq. (24)). In Fig. 7 variation of effective potential for BI photons as compared to Maxwellian photon are shown. For plotting, we use Eqs. [5452] 33. Similar to the $\alpha=1$ special case, we consider $\bar{r} \in\left[\bar{r}_{t h}, \infty\right]$, where, $\bar{r}_{t h}^{2}=\sqrt{\kappa q^{2} / \alpha}[2+\alpha(\sqrt{2}-1)]$, for a range of $\alpha$. From the plots in Fig. 7 it is evident that the radius of the photon sphere $\left(r_{p s}\right)$ for BI photons is smaller than that of Maxwell photons (irrespective of positive or, negative $\kappa$ ). Furthermore, the photon sphere decreases as the values of $b^{2}$ decreases.

Let us now write the expression for the deflection of light (BI photon) from the null geodesic equation for the effective metric given by Eq. (511). We get the expression for the deflection angle as,

$$
\Delta \phi=2 \int_{\bar{r}_{t p}}^{\infty} \frac{U(\bar{r})}{r^{2}(\bar{r})}\left[\frac{U\left(\bar{r}_{t p}\right) e^{2 \psi\left(\bar{r}_{t p}\right)}}{r^{2}\left(\bar{r}_{t p}\right)}-\frac{U(\bar{r}) e^{2 \psi(\bar{r})}}{r^{2}(\bar{r})}\right]^{-1 / 2} d \bar{r}-\pi
$$




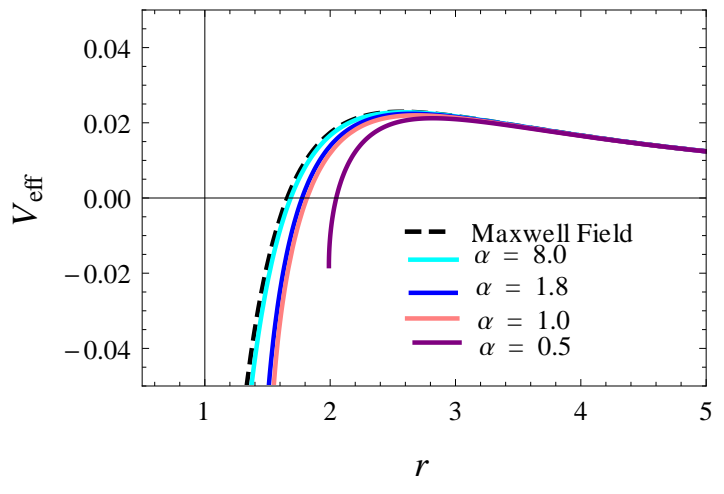

(a) $\kappa=1.0, L=1.0, q=0.7, M=1.0$

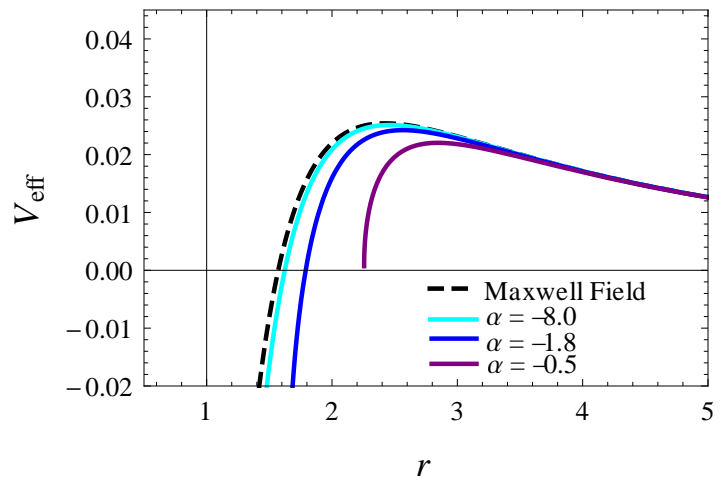

(b) $\kappa=-1.0, L=1.0, q=0.9, M=1.0$

FIG. 7. Effective potential $\left(V_{e f f}\right)$ for BI photons are plotted and compared with that of the Maxwellian photon (black dashed line) propagating in the background of EiBI spacetimes. In both plots, $\kappa$ value is same (but of opposite sign). However, $\alpha=4 \kappa b^{2}$ takes different vaues in the plots.

where, $r^{2}(\bar{r})$ is the physical radial distance square given by Eq. (53) and $\bar{r}_{t p}$ is the coordinate value corresponding to the physical radial distance at the turning point $\left(r_{t p}\right)$. Again, for the Maxwell photon, we get the deflection angle,

$$
\Delta \phi^{\operatorname{Max}}=2 \int_{r_{t p}}^{\infty} \frac{1}{\sqrt{r^{4}+\kappa q^{2}}}\left[\frac{r_{t p}^{2} h_{1}\left(r_{t p}\right)}{r_{t p}^{4}-\kappa q^{2}}-\frac{r^{2} h_{1}(r)}{r^{4}-\kappa q^{2}}\right]^{-1 / 2} d r-\pi
$$

where (56) is expressed directly in terms of physical radial distance $(r)$. In Fig. 8 the variation of the deflection angle of light as function of $r_{t p}$ is shown for different $\alpha$ values and it is compared with that for the Maxwell field. To visualize the correspondence between the effective potential for photons with the deflection angle of light, same parameter values are used in the plots. For plotting, Eqs. 55, 56, 39] are used.

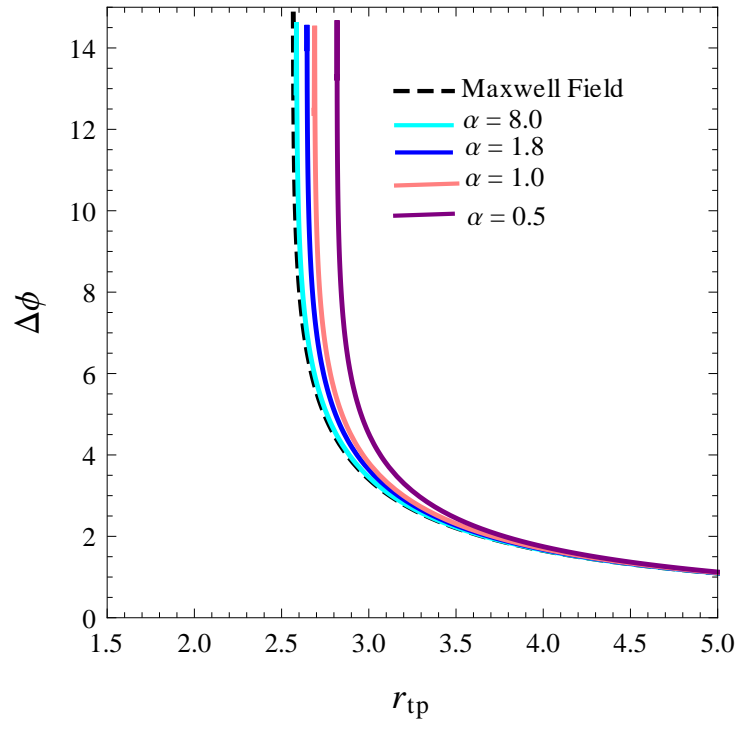

(a) $\kappa=1.0, L=1.0, q=0.7, M=1.0$

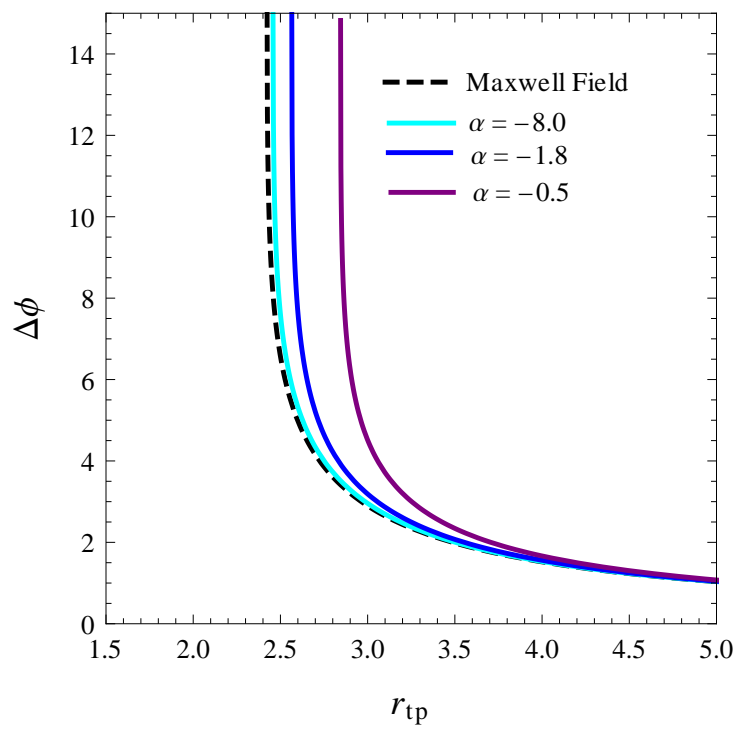

(b) $\kappa=-1.0, L=1.0, q=0.9, M=1.0$

FIG. 8. Deflection angle $(\Delta \phi)$ for BI photons are plotted as a function of the physical radial distance at the turning point $\left(r_{t p}\right)$ and compared with that of the Maxwellian photon (black dashed line) propagating in the background of EiBI spacetimes. The deflection angle diverges rapidly when the turning-point-radius $\left(r_{t p}\right)$ becomes closer to the radius of the photon sphere $\left(r_{p s}\right)$. In both plots, the parameter values are the same as in Fig. 7

From the plots of both the effective potential (Fig. 7) and strong deflection angle (Fig. 87), we note that with the increase of the value of $b^{2}$, the radius of the photon sphere $\left(r_{p s}\right)$ decreases. 


\section{CONCLUSIONS}

In this article, we have derived new classes of spherically symmetric static solutions in EiBI gravity coupled to Born-Infeld electrodynamics. The spacetimes demonstrate the effect of coupling a non-linear electrodynamics (BornInfeld electrodynamics) to a modified theory of gravity (the EiBI gravity). All solutions are analytical. We summarize our results below, point wise:

(i) We have used a non-standard choice for the physical and auxiliary metrics to find the spacetimes in EiBI gravity. The standard ansatze for the physical and auxiliary line elements as employed in [8, 40 42], led to a complicated set of coupled differential equations. We note that our non-standard choice is far more useful insofar as solution construction is concerned. Similar unconventional choices may be used in other cases with the hope of simplifying the set of equations we need to solve.

(ii) We obtain two classes of spacetimes in section III. All the known results for different electrically charged, spherically symmetric, static scenarios can be obtained by taking different limits of $\alpha=4 \kappa b^{2}$ and $\kappa$. In general, the spacetimes we obtain are singular at the location of the charge, where the charge can be localized about a point or distributed over a spherical shell (2-sphere). The singularity may or may not be covered by an event horizon, i.e. it may be a black hole or a naked singularity. However, all solutions converge to the Reissner-Nordström solution at large distances.

(iii) The effects of modifications in both gravity and matter sectors are demonstrated through the analysis of the metric functions for fixed $b^{2}$, varying $\kappa$ and vice-versa. In our analysis, we find some interesting features. For a fixed $\kappa$, the horizon radius increases with the decrease of $b^{2}$ for some parameter values $(\kappa, q, M)$. If $b^{2}$ is fixed, the horizon radius decreases with increasing $\kappa(\kappa>0)$.

(iv) Although the results are analytical, the expressions for the metric functions are complicated. However, a special choice, $\alpha=1(\kappa>0)$, leads to a simple solution that looks similar to the Reissner-Nordström. We analyze this special solution in detail in section IV. We find that this spacetime also possesses the same generic features mentioned above in (ii). Subsequently, for the $\alpha=1$ line element, we investigate the null geodesics in the effective geometry for BI photons, as well as gravitational lensing. We find that as $\kappa$ is increased, the radius of the photon sphere increases. We also find the leading order contribution of $\kappa$ in the expression for the weak deflection angle. The overall sign of this term is positive but it depends on the value of $\kappa$.

$(v)$ In section $\mathrm{V}$ we investigate the effective potential, photon sphere, and strong deflection angle for $\alpha \neq 1$, through numerics and plots. We compare with results for a Maxwellian electric field. Analyzing the plots, we find that for a fix value of $\kappa$ (irrespective of its sign), the radius of the photon sphere decreases as the value of $b^{2}$ is increased.

In summary, using non-standard ansatze for the physical and auxiliary metrics, we have been able to obtain analytical solutions in Born-Infeld gravity coupled to Born-Infeld electrodynamics. One may explore strong gravitational lensing further using the approach in [63]. It is possible that there are other non-singular solutions as well which are nonsingular from both the gravitational and electrodynamic perspectives. For this, it is essential to scan the $\left(\kappa, b^{2}, \frac{q}{M}\right)$ parameter space more thoroughly. In [43], the author has shown that in the case of EiBI solutions with a Maxwellian electric field, there exists a region in the parameter space $(\kappa, q, M)$ which allows the construction of a Lorentzian wormhole without violating the weak or null energy conditions. In our solution, a similar analysis is possible. One can also study the black hole thermodyanmics using the solutions presented here. We hope to work on these issues and communicate our results in the near future.

\section{ACKNOWLEDGMENTS}

The authors thank Jonas P. Pereira for pointing out a crucial fact on light propagation in nonlinear electrodynamics and also for his comments.

\section{Appendix: Integral results}

Here, we compute the integrals $\int V(\bar{r}) \bar{r}^{2} d \bar{r}$, in detail, which have been used in the section पIIto obtain the analytical expressions of two different classes of spacetimes [Eq. (19) and Eq. (21)]. These integrals are non-trivial and may not be computed correctly otherwise (e.g. using Mathematica). 
$(a) \infty>\alpha>1$ case:

$$
\int V \bar{r}^{2} d \bar{r}=-\frac{(2-\alpha) \bar{r}^{3}}{6(\alpha-1)}+\frac{\alpha}{2(\alpha-1)} \int \sqrt{\bar{r}^{4}-\frac{4 \kappa q^{2}(\alpha-1)}{\alpha}} d \bar{r}
$$

The last term on the R.H.S. of the Eq. A.1 is a non-trivial integral which we evaluate below:

$$
\begin{aligned}
\int \sqrt{\bar{r}^{4}-\beta} d \bar{r} & =\bar{r} \sqrt{\bar{r}^{4}-\beta}-\int \frac{2 \bar{r}^{4} d \bar{r}}{\sqrt{\bar{r}^{4}-\beta}}, \quad \text { ( Using partial integration) } \\
& =\bar{r} \sqrt{\bar{r}^{4}-\beta}-2 \int \sqrt{\bar{r}^{4}-\beta} d \bar{r}-2 \beta \int \frac{d \bar{r}}{\sqrt{\bar{r}^{4}-\beta}} \\
& =\frac{1}{3} \bar{r} \sqrt{\bar{r}^{4}-\beta}-\frac{2 \beta^{3 / 4}}{3} \int \frac{d y}{\sqrt{y^{4}-1}}, \quad\left(\bar{r}=\beta^{1 / 4} y\right) \\
\text { Now, } \quad \int \frac{d y}{\sqrt{y^{4}-1}} & =-\int \frac{d u}{\sqrt{1-u^{4}}}, \quad(u=1 / y) \\
& =-F(\arcsin (1 / y) \mid-1)+C_{\text {ext }} \\
\text { So, } \quad \int \sqrt{\bar{r}^{4}-\beta} d \bar{r} & =\frac{1}{3} \bar{r} \sqrt{\bar{r}^{4}-\beta}+\frac{2 \beta^{3 / 4}}{3}\left(F\left(\arcsin \left(\beta^{1 / 4} / \bar{r}\right) \mid-1\right)-C_{e x t}\right)
\end{aligned}
$$

where, $F(\phi \mid m)=\int_{0}^{\phi}\left[1-m \sin ^{2} \theta\right]^{-1 / 2} d \theta$ is the incomplete elliptic integral of the first kind. Here, $\beta=\frac{4 \kappa q^{2}(\alpha-1)}{\alpha}$ $(\beta>0)$ and $C_{\text {ext }}$ is an additional constant term appearing due to the fact that Elliptic integrals are defined with the zero lower limit and hence, these are not the so-called indefinite integrals. The Eq. A.1) becomes,

$$
\begin{aligned}
\int V \bar{r}^{2} d \bar{r}=\frac{\bar{r}^{3}}{3}+ & \frac{\alpha \bar{r}^{3}}{6(\alpha-1)}\left[\sqrt{1-\frac{4 \kappa q^{2}(\alpha-1)}{\alpha \bar{r}^{4}}}-1\right]+ \\
& \frac{\alpha^{1 / 4}\left(4 \kappa q^{2}\right)^{3 / 4}}{3(\alpha-1)^{1 / 4}}\left[F\left(\arcsin \left(\frac{\left(4 \kappa q^{2}(\alpha-1)\right)^{1 / 4}}{\alpha^{1 / 4} \bar{r}}\right) \mid-1\right)-C_{\text {ext }}\right]
\end{aligned}
$$

Now, to fix up the extra constant $C_{\text {ext }}$, we use the fact that, in the limit of $\alpha \rightarrow \infty$ and $\kappa \rightarrow 0$, the full expression of $e^{2 \psi}$ must take form of the Reissner-Nordström solution (i.e. $e^{2 \psi} \rightarrow 1-\frac{2 M}{\bar{r}}+\frac{q^{2}}{\bar{r}^{2}}$ ). Under this constraint, $C_{\text {ext }}=0$.

$$
\text { (b) }-\infty<\alpha<1 \text { case: }
$$

$$
\int V \bar{r}^{2} d \bar{r}=-\frac{(2-\alpha) \bar{r}^{3}}{6(\alpha-1)}+\frac{\alpha}{2(\alpha-1)} \int \sqrt{\bar{r}^{4}+\gamma} d \bar{r}
$$

where, $\gamma=\frac{4 \kappa q^{2}(1-\alpha)}{\alpha}(\gamma>0)$. We evaluate the last integral on R.H.S. of the Eq. (A.4) below:

$$
\begin{aligned}
\int \sqrt{\bar{r}^{4}+\gamma} d \bar{r} & =\frac{1}{3} r \sqrt{\bar{r}^{4}+\gamma}+\frac{2 \gamma^{3 / 4}}{3} \int \frac{d y}{\sqrt{y^{4}+1}}, \quad\left(\bar{r}=\gamma^{1 / 4} y\right) \\
\text { Now, } \quad \int \frac{d y}{\sqrt{y^{4}+1}} & =-\sqrt{i} F(i \operatorname{arcsinh}(\sqrt{i} y) \mid-1)+C_{e x t}^{\prime} \\
\text { So, } \quad \int \sqrt{\bar{r}^{4}+\gamma} d \bar{r} & =\frac{1}{3} \bar{r} \sqrt{\bar{r}^{4}+\gamma}-\frac{2 \gamma^{3 / 4}}{3}\left[\sqrt{i} F\left(i \operatorname{arcsinh}\left(\sqrt{i} r \gamma^{-1 / 4}\right) \mid-1\right)-C_{e x t}^{\prime}\right]
\end{aligned}
$$

where, $i=\sqrt{-1}$. So, the Eq. A.4 becomes

$$
\begin{aligned}
\int V \bar{r}^{2} d \bar{r}= & \frac{\bar{r}^{3}}{3}+\frac{\alpha \bar{r}^{3}}{6(\alpha-1)}\left[\sqrt{1+\frac{4 \kappa q^{2}(1-\alpha)}{\alpha \bar{r}^{4}}}-1\right] \\
& +\frac{\alpha^{1 / 4}\left(4 \kappa q^{2}\right)^{3 / 4}}{3(1-\alpha)^{1 / 4}}\left[\sqrt{i} F\left(i \operatorname{arcsinh}\left(\frac{\sqrt{i} \alpha^{1 / 4} \bar{r}}{\left(4 \kappa q^{2}(1-\alpha)\right)^{1 / 4}}\right) \mid-1\right)-C_{e x t}^{\prime}\right]
\end{aligned}
$$


Similar to the earlier case, we fix up the extra constant $C_{\text {ext }}^{\prime}$, by taking the limit of $\alpha \rightarrow-\infty$ and $\kappa \rightarrow-0$. Then also, the full expression of $e^{2 \psi}$ takes the form of the Reissner-Nordström solution. Under this constraint, $C_{e x t}^{\prime}=-\int_{0}^{\infty} \frac{d y}{\sqrt{y^{4}+1}}=-\frac{\Gamma^{2}(1 / 4)}{4 \sqrt{\pi}}$. Eq. A.6. can be rewritten further as,

$$
\int V \bar{r}^{2} d \bar{r}=\frac{\bar{r}^{3}}{3}+\frac{\alpha \bar{r}^{3}}{6(\alpha-1)}\left[\sqrt{1+\frac{4 \kappa q^{2}(1-\alpha)}{\alpha \bar{r}^{4}}}-1\right]+\left(\frac{4 \kappa q^{2}}{3 \bar{r}}\right){ }_{2} F_{1}\left(\frac{1}{4}, \frac{1}{2} ; \frac{5}{4} ;-\frac{4 \kappa q^{2}(1-\alpha)}{\alpha \bar{r}^{4}}\right)
$$

where, we use

$$
\sqrt{i} F(i \operatorname{arcsinh}(\sqrt{i} y) \mid-1)+\frac{\Gamma^{2}(1 / 4)}{4 \sqrt{\pi}}=\int_{y}^{\infty} \frac{d z}{\sqrt{z^{4}+1}}=\frac{{ }_{2} F_{1}\left(\frac{1}{4}, \frac{1}{2} ; \frac{5}{4} ;-\frac{1}{y^{4}}\right)}{y} .
$$

Here, ${ }_{2} F_{1}(a, b ; c ; z)$ is the hypergeometric function with usual meaning.

[1] S. W. Hawking and G. F. R. Ellis, The Large Scale Structure of Space-Time (Cambridge University Press, Cambridge, England, 1975)

[2] A. Eddington, The Mathematical Theory of Relativity (Cambridge University Press, Cambridge, England, 1924)

[3] M. Born and L. Infeld, Proc. R. Soc. A 144, 425 (1934)

[4] S. Deser and G. W. Gibbons, Classical and Quantum Gravity 15, L35 (1998)

[5] D. N. Vollick, Phys. Rev. D 69, 064030 (2004)

[6] D. N. Vollick, Phys. Rev. D 72, 084026 (2005)

[7] D. N. Vollick, arXiv:gr-qc/0601136

[8] M. Bañados and P. G. Ferreira, Phys. Rev. Lett. 105, 011101 (2010)

[9] C. J. Isham, A. Salam, and J. Strathdee, Phys. Rev. D 3, 867 (1971)

[10] J. H. C. Scargill, M. Banados, and P. G. Ferreira, Phys. Rev. D 86, 103533 (2012)

[11] A. Schmidt-May and M. von $\operatorname{Strauss}(2014)$, arXiv:1412.3812 [hep-th]

[12] J. B. Jimnez, L. Heisenberg, and G. J. Olmo, Journal of Cosmology and Astroparticle Physics 2014, 004 (2014)

[13] P. Pani, V. Cardoso, and T. Delsate, Phys. Rev. Lett. 107, 031101 (2011)

[14] J. Casanellas, P. Pani, I. Lopes, and V. Cardoso, The Astrophysical Journal 745, 15 (2012)

[15] P. P. Avelino, Phys. Rev. D 85, 104053 (2012)

[16] Y.-H. Sham, L.-M. Lin, and P. T. Leung, Phys. Rev. D 86, 064015 (2012)

[17] Y.-H. Sham, P. T. Leung, and L.-M. Lin, Phys. Rev. D 87, 061503 (2013)

[18] T. Harko, F. S. N. Lobo, M. K. Mak, and S. V. Sushkov, Phys. Rev. D 88, 044032 (2013)

[19] H. Sotani, Phys. Rev. D 89, 104005 (2014)

[20] H. Sotani, Phys. Rev. D 89, 124037 (2014)

[21] H. Sotani(2015), arXiv:1503.07942 [astro-ph.HE]

[22] C. Escamilla-Rivera, M. Banados, and P. G. Ferreira, Phys. Rev. D 85, 087302 (2012)

[23] I. Cho, H.-C. Kim, and T. Moon, Phys. Rev. D 86, 084018 (2012)

[24] P. P. Avelino and R. Z. Ferreira, Phys. Rev. D 86, 041501 (2012)

[25] A. De Felice, B. Gumjudpai, and S. Jhingan, Phys. Rev. D 86, 043525 (2012)

[26] K. Yang, X.-L. Du, and Y.-X. Liu, Phys. Rev. D 88, 124037 (2013)

[27] M. Lagos, M. Bañados, P. G. Ferreira, and S. Garcia-Saenz, Phys. Rev. D 89, 024034 (2014)

[28] X.-L. Du, K. Yang, X.-H. Meng, and Y.-X. Liu, Phys. Rev. D 90, 044054 (2014)

[29] T. Harko, F. S. Lobo, and M. K. Mak, Galaxies 2, 496 (2014)

[30] Y.-X. Liu, K. Yang, H. Guo, and Y. Zhong, Phys. Rev. D 85, 124053 (2012)

[31] T. Delsate and J. Steinhoff, Phys. Rev. Lett. 109, 021101 (2012)

[32] I. Cho and H.-C. Kim, Phys. Rev. D 88, 064038 (2013)

[33] S. Jana and S. Kar, Phys. Rev. D 88, 024013 (2013)

[34] J. Casanellas, P. Pani, I. Lopes, and V. Cardoso, The Astrophysical Journal 745, 15 (2012)

[35] P. Avelino, Journal of Cosmology and Astroparticle Physics 2012, 022 (2012)

[36] P. Pani and T. P. Sotiriou, Phys. Rev. Lett. 109, 251102 (2012)

[37] H.-C. Kim, Phys. Rev. D 89, 064001 (2014)

[38] S. D. Odintsov, G. J. Olmo, and D. Rubiera-Garcia, Phys. Rev. D 90, 044003 (2014)

[39] K. Fernandes and A. Lahiri, Phys. Rev. D 91, 044014 (2015)

[40] S.-W. Wei, K. Yang, and Y.-X. Liu(2014), arXiv:1405.2178 [gr-qc]

[41] H. Sotani and U. Miyamoto, Phys. Rev. D 90, 124087 (2014)

[42] G. J. Olmo, D. Rubiera-Garcia, and H. Sanchis-Alepuz, The European Physical Journal C 74, 2804 (2014)

[43] R. Shaikh, Phys. Rev. D 92, 024015 (2015) 
[44] M. Demianski, Foundations of Physics 16, 187 (1986)

[45] T. Tamaki and T. Torii, Phys. Rev. D 62, 061501 (2000)

[46] R. Yamazaki and D. Ida, Phys. Rev. D 64, 024009 (2001)

[47] S. H. Mazharimousavi and M. Halilsoy(2014), arXiv:1405.2956 [gr-qc]

[48] R. Banerjee and D. Roychowdhury, Phys. Rev. D 85, 104043 (2012)

[49] A. Lala and D. Roychowdhury, Phys. Rev. D 86, 084027 (2012)

[50] R. Banerjee and D. Roychowdhury, Phys. Rev. D 85, 044040 (2012)

[51] J. P. Pereira and J. A. Rueda, Phys. Rev. D 91, 064048 (2015)

[52] E. Fradkin and A. Tseytlin, Physics Letters B 163, 123 (1985)

[53] A. Abouelsaood, C. Callan, C. Nappi, and S. Yost, Nuclear Physics B 280, 599 (1987)

[54] R. M. Wald, General Relativity (University of Chicago Press, Chacago and London, 1984)

[55] J. F. Plebansky, Lectures on Nonlinear Electrodynamics (Nordita, Copenhagen, 1968)

[56] M. Novello, V. A. De Lorenci, J. M. Salim, and R. Klippert, Phys. Rev. D 61, 045001 (2000)

[57] C.-M. Claudel, K. S. Virbhadra, and G. F. R. Ellis, JOURNAL OF MATHEMATICAL PHYSICS 42, 2 (2001)

[58] K. S. Virbhadra and G. F. R. Ellis, Phys. Rev. D 65, 103004 (2002)

[59] K. S. Virbhadra, Phys. Rev. D 79, 083004 (2009)

[60] K. S. Virbhadra and G. F. R. Ellis, Phys. Rev. D 62, 084003 (2000)

[61] J. Bodenner and C. M. Will, American Journal of Physics 71, 770 (2003)

[62] J. Briet and D. Hobill(2008), arXiv:0801.3859 [gr-qc]

[63] V. Bozza, S. Capozziello, G. Iovane, and G. Scarpetta, General Relativity and Gravitation 33, 1535 (2001) 